\title{
Laplacian Eigenfunctions in NMR. I. A Numerical Tool
}

\author{
DENIS S. GREBENKOV
}

Laboratoire de Physique de la Matière Condensée, CNRS - Ecole Polytechnique, Palaiseau F-91128, France

\begin{abstract}
A geometrical confinement considerably affects the diffusive motion of the nuclei and the consequent signal attenuation under inhomogeneous magnetic fields. In this article, we illustrate the use of Laplacian eigenfunctions to describe this effect. Starting from the classical Bloch-Torrey equation, we obtain the free induction decay (FID) and the spin-echo or gradient-echo signal in a compact matrix form. Each attenuation mechanism (restricted diffusion, gradient dephasing, surface or bulk relaxation) is represented by a matrix which is constructed from the Laplace operator eigenbasis and thus depending only on the geometry of the confinement. In turn, the physical parameters (free diffusion coefficient, gradient intensity, surface or bulk relaxivity) characterize the "strengths" of the underlying attenuation mechanisms and naturally appear as coefficients in front of these matrices. Once the Laplacian eigenfunctions for a given confinement are found (analytically or numerically), further computation of the macroscopic signal is more accurate and much faster than by using conventional simulation methods. The matrix technique is actually a simple numerical tool to deal with arbitrary gradient waveforms, including simple or stimulated, single or multiple spin echoes. We illustrate its efficiency by considering restricted diffusion in simple domains: a slab, a cylinder, and a sphere. In a companion paper, we shall focus on theoretical advances achieved by using Laplacian eigenfunctions. (c) 2008 Wiley Periodicals, Inc. Concepts Magn Reson Part A 32A: 277-301, 2008.
\end{abstract}

KEY WORDS: Laplace operator; eigenfunction; eigenvalue; restricted diffusion; (reflected) Brownian motion; spin echo; gradient echo; confinement; NMR; matrix formalism; Bloch-Torrey equation

\section{INTRODUCTION}

In this article, we discuss recent advances in understanding restricted diffusion and its role in nuclear

\footnotetext{
Received 6 March 2008; revised 8 May 2008; accepted 20 May 2008

Correspondence to: Denis S. Grebenkov. E-mail: denis.grebenkov@ polytechnique.edu

Concepts in Magnetic Resonance Part A, Vol. 32A(4) 277-301 (2008) Published online in Wiley InterScience (www.interscience.wiley. com). DOI 10.1002/cmr.a.20117

(c) 2008 Wiley Periodicals, Inc.
}

magnetic resonance (NMR). A special focus is on Laplacian eigenfunctions and their use in theoretical and numerical studies of diffusive processes. Our aim is to provide an introduction, as simple as possible, to the related mathematical and physical concepts which often appear in modern NMR literature. Whenever possible, the discussion remains at intuitive level, with many illustrations and examples. This article is a pedagogical complementary to the recent review (I) containing further mathematical details and numerous references, as well as a historical overview.

The focus on Laplacian eigenfunctions, containing exhaustive information about restricted diffusion and 
its geometrical confinement, is not accidental. Figuratively speaking, Laplacian eigenfunctions form the "alphabet" of the proper mathematical language describing restricted diffusion. Instead of searching for one particular solution of a diffusion problem, one aims to get all the solutions at once. Of course, this is a more difficult task, but this task has to be solved only once for a given confinement. When this preliminary step is accomplished, further analysis (e.g., the computation of the spin-echo signal) is more accurate and much faster than by using conventional simulation methods. This "spectral" point of view is probably not shared by many NMR practitioners who are more familiar with averaged propagator and the related $q$-analysis. In fact, after a brief glance through the article, the presented matrix approach may appear as a sophisticated abstract formalism. The author's ambition is to convince the reader that the spectral mathematics beyond the classical Fourier transform of the averaged propagator is as easy and is actually more efficient in many aspects.

The content is split in two parts which are devoted respectively to numerical and theoretical issues. In the next section, we start with a reminder of the basic facts on restricted diffusion. In particular, the notion of Laplacian eigenfunctions is illustrated by many examples. In Section III, we provide a mathematical basis for an efficient numerical tool by deriving simple matrix expressions for the macroscopic signal. The signal is determined by three governing matrices $\Lambda, \mathcal{B}$, and $\tilde{\mathcal{B}}^{s}$, representing the evolution of the magnetization due to diffusion, gradient dephasing, and surface relaxation, respectively. In Section IV, we explain the implementation of this numerical tool for simple domains: a slab, a cylinder, and a sphere. In a companion paper (2), we shall focus on theoretical advances which could be achieved by using Laplacian eigenfunctions.

The splitting in two parts gives us an opportunity to present the concepts of restricted diffusion from two alternative points of view. In the first part, we adopt the "macroscopic" deterministic approach based on the Bloch-Torrey equation describing the evolution of the magnetization. This quantity already represents the contribution of a large number of diffusing particles so that all the randomness is averaged out. In a companion paper, we shall employ the "microscopic" probabilistic description through the reflected Brownian motion (3). In this case, one studies individual random trajectories of the diffusing particles and the related dephasing in a magnetic field. The macroscopic characteristics such as the moments of the dephasing are then determined by averaging. Although these two descriptions are equivalent, the first one is appropriate for numerical computation (simple expressions for the signal), while the second one is natural for theoretical analysis. These two insights on restricted diffusion are complementary and helpful.

\section{BASIC FACTS ON DIFFUSION}

In this section, we remind the basic facts about diffusion: schematic "derivation" of diffusion (or heat) equation and introduction of the diffusive propagator (Section "Diffusion Equation"); discussion of boundary conditions (Section "Boundary Condition"); magnetic encoding and Bloch-Torrey equation (Section "Magnetic Field Encoding"); eigenfunctions and eigenvalues of the Laplace operator (Section "Notion of Eigenfunction"). The familiar reader can skip this section.

\section{Diffusion Equation}

Diffusion is a fundamental transport mechanism, with countless examples in nature and applications in sciences, from physics to biology, chemistry, engineering, and economics. Although the microscopic dynamics of atoms, molecules, or other species with short-range interactions may be defiantly complex, its coarser description via diffusion equation at macroscopic time and length scales is often appropriate and very accurate. At these scales, a small macroscopic volume around each bulk point $\mathbf{r}$ contains a large number of particles, allowing one to introduce a density $c(\mathbf{r}, t)$. The specific motional features of the complex microscopic dynamics are averaged out, and the time evolution of the macroscopic density is governed by a diffusion equation.

Phenomenological Derivation. If no particle is created or destroyed in the bulk, the time evolution of the density of particles $c(\mathbf{r}, t)$ is described by the continuity equation

$$
\frac{\partial c(\mathbf{r}, t)}{\partial t}+\nabla \mathbf{j}(\mathbf{r}, t)=0
$$

The second term, the divergence of the flux density $\mathbf{j}(\mathbf{r}, t)$, is the scalar product between gradient operator $\nabla$ and the vector $\mathbf{j}(\mathbf{r}, t)=\left(j_{1}(\mathbf{r}, t), \ldots\right.$, $\left.j_{d}(\mathbf{r}, t)\right)$ :

$$
\nabla \mathbf{j}(\mathbf{r}, t)=\frac{\partial}{\partial x_{1}} j_{1}(\mathbf{r}, t)+\cdots+\frac{\partial}{\partial x_{d}} j_{d}(\mathbf{r}, t),
$$


$d$ being the dimension of space, and $\partial / \partial x_{k}$ the derivative with respect to the $k$ th spatial coordinate. This term describes inflow and outflow of particles into and out of a vicinity of a bulk point $\mathbf{r}$ at time $t$. In other words, the continuity equation states that the density $c(\mathbf{r}, t)$ evolves in time only because of the particles coming to and leaving the point $\mathbf{r}$. This is a general relation.

On the other hand, the particles diffuse to diminish the gradient of their density $c(\mathbf{r}, t)$. This tendency is mathematically represented by the phenomenological Fick's law

$$
\mathbf{j}(\mathbf{r}, t)=-D \nabla c(\mathbf{r}, t)
$$

Throughout the paper, the free diffusion coefficient $D$ is assumed to be constant (no dependence on time and spatial coordinates). Substituting $\mathbf{j}(\mathbf{r}, t)$ into the continuity equation, one gets the diffusion (or heat) equation

$$
\frac{\partial}{\partial t} c(\mathbf{r}, t)-D \Delta c(\mathbf{r}, t)=0
$$

where $\Delta=\nabla^{2}=\partial^{2} / \partial x_{1}^{2}+\cdots+\partial^{2} / \partial x_{d}^{2}$ is the Laplace operator.

Local Dynamics. The above phenomenological "derivation" can be replaced by more subtle analysis of the local dynamics. During a short time $\tau$, particles travel on average a typical distance $\sigma$. While the particles from a point $\mathbf{r}$ move away, the particles from neighboring points come to $\mathbf{r}$. This migration is intended to level off the gradient of the density $c(\mathbf{r}, t)$. To illustrate the idea, we simplify the picture by allowing the particles to move only along $2 d$ preferential directions in space. In other words, we consider the migration between the point $\mathbf{r}$ and its $2 d$ neighbors $\mathbf{r}_{i}$ at distance $\sigma$. If the motion is isotropic, each particle from each neighboring site can move to $\mathbf{r}$ with probability $(2 d)^{-1}$. The density of particles at $\mathbf{r}$ at time $t+\tau$ is then constituted of all the particles coming to $\mathbf{r}$ :

$$
c(\mathbf{r}, t+\tau)=\frac{1}{2 d} \sum_{i=1}^{2 d} c\left(\mathbf{r}_{i}, t\right) .
$$

Subtracting $c(\mathbf{r}, t)$ and dividing by $\tau$ yield

$$
\underbrace{\frac{c(\mathbf{r}, t+\tau)-c(\mathbf{r}, t)}{\tau}}_{\partial c(\mathbf{r}, t) / \partial t}=\underbrace{\frac{\sigma^{2}}{2 d \tau}}_{D} \underbrace{\sum_{i=1}^{2 d} \frac{c\left(\mathbf{r}_{i}, t\right)-c(\mathbf{r}, t)}{\sigma^{2}}}_{\Delta c(\mathbf{r}, t)}
$$

In the limit of vanishing $\tau$ and $\sigma^{2}$, the left-hand side becomes the time derivative of the density, while the sum approximates the Laplace operator acting on $c(\mathbf{r}, t)$. The ratio between two microscopic transport parameters $\sigma^{2}$ and $\tau$ is the free diffusion coefficient $D$. Although the above "derivation" of diffusion equation is simplified, the result is very general. For instance, one could easily avoid the introduction of "preferential directions" by considering the particles coming from a sphere of radius $\sigma$ around $\mathbf{r}$. At first thought it may sound paradoxical to speak simultaneously about random diffusive motion and deterministic diffusion equation. It is worth noting that, although each individual trajectory of the diffusing particle is random, the density is a macroscopic quantity obtained by averaging over a large number of particles.

The local character of the microscopic dynamics is a crucial assumption for deriving the diffusion equation. If very large moves were allowed during a short time, the density $c(\mathbf{r}, t+\tau)$ would be constituted of particles coming from neighboring and distant regions. In this case, the sum in Eq. [3] would include distant points and converge to an integral over the bulk. This would be a spatially nonlocal macroscopic dynamics. On the other hand, if the microscopic moves were highly correlated, the density at time $t+\tau$ would depend not only on the previous moment $t$, but on the whole preceding evolution. In this case, one would deal with a temporarily nonlocal macroscopic dynamics. Although both situations are often encountered in practice, we do not consider such anomalous diffusions in this paper [see (4-9) and references therein].

Diffusive Propagator. The diffusion equation [2] describes the evolution of the density from an initial state. Specifying the initial density of particles $\rho\left(\mathbf{r}_{0}\right)$ at time $t=0$, one fixes the solution of Eq. [2] and the whole evolution of the system. Among various initial conditions, a point-like source plays a special role. In fact, it is natural to ask how does the density of particles started from a given point $\mathbf{r}_{0}$ evolve? Throughout this paper, $G_{t}\left(\mathbf{r}_{0}, \mathbf{r}\right)$ denotes the family of these densities, parametrized by the starting point $\mathbf{r}_{0}$, that satisfy Eq. [2] with respect to the arrival point $\mathbf{r}$. The initial condition for all particles concentrated in one point $\mathbf{r}_{0}$ is mathematically represented by the Dirac distribution:

$$
G_{t=0}\left(\mathbf{r}_{0}, \mathbf{r}\right)=\delta\left(\mathbf{r}-\mathbf{r}_{0}\right)
$$

As it will be shown later, the function $G_{t}\left(\mathbf{r}_{0}, \mathbf{r}\right)$ is symmetric with respect to interchange of $\mathbf{r}_{0}$ and $\mathbf{r}$ : 


$$
G_{t}\left(\mathbf{r}_{0}, \mathbf{r}\right)=G_{t}\left(\mathbf{r}, \mathbf{r}_{0}\right) .
$$

Depending on the application field, the solution $G_{t}\left(\mathbf{r}_{0}, \mathbf{r}\right)$ of Eqs. [2] and [4] bears different names: diffusive propagator, heat kernel, or Green function of diffusion equation. This is an elementary "block" describing the dynamics of particles at macroscopic level. As such, the propagator contains all available information about diffusion. In particular, a solution $c(\mathbf{r}, t)$ of the diffusion equation [2] with a given initial density $\rho\left(\mathbf{r}_{0}\right)$ is simply expressed through the propagator:

$$
c(\mathbf{r}, t)=\int d \mathbf{r}_{0} \rho\left(\mathbf{r}_{0}\right) G_{t}\left(\mathbf{r}_{0}, \mathbf{r}\right)
$$

(the integral here is over the whole space). This formula gives two complementary insights on the propagator. From the physical point of view, the initial density $\rho\left(\mathbf{r}_{0}\right)$ describes a collection of independent point-like sources of particles distributed in space at time $t=0$. Each of them evolves in time, independently of the others, with the propagator $G_{t}\left(\mathbf{r}_{0}, \mathbf{r}\right)$ for the particles started at $\mathbf{r}_{0}$. The density $c(\mathbf{r}, t)$ is then a linear superposition (represented here by the integral) of the resulting densities $\rho\left(\mathbf{r}_{0}\right) G_{t}\left(\mathbf{r}_{0}, \mathbf{r}\right)$ for each point $\mathbf{r}_{0}$. From the probabilistic point of view, one can think of a single particle whose initial location in space is random, with a given probability density $\rho\left(\mathbf{r}_{0}\right)$ (normalized to 1$)$. In this language, the starting point is chosen at time $t=0$ with probability $\rho\left(\mathbf{r}_{0}\right) d \mathbf{r}_{0}$, and then the particle diffuses up to time $t$, the integral averaging over all starting points $\mathbf{r}_{0}$. The solution $c(\mathbf{r}, t)$ is then the probability density to find this particle at time $t(10)$.

Example: Gaussian Propagator. The diffusive propagator for the whole space has a simple analytical form

$$
G_{t}^{\text {space }}\left(\mathbf{r}_{0}, \mathbf{r}\right)=(4 \pi D t)^{-d / 2} \exp \left[\frac{-\left(\mathbf{r}-\mathbf{r}_{0}\right)^{2}}{4 D t}\right] .
$$

It is easy to check that this function satisfies the diffusion equation [2]. When $t$ goes to 0 , the density $G_{t}^{\text {space }}\left(\mathbf{r}_{0}, \mathbf{r}\right)$ vanishes for $\mathbf{r} \neq \mathbf{r}_{0}$ (particles have no enough time to diffuse from $\mathbf{r}_{0}$ to $\mathbf{r}$ ), and diverges for $\mathbf{r}=\mathbf{r}_{0}$ (particles remain concentrated in an infinitely small volume). In fact, $G_{t}^{\text {space }}\left(\mathbf{r}_{0}, \mathbf{r}\right)$ approaches the Dirac distribution and thus satisfies the initial condition [4]. The explicit form of the diffusive propagator in the whole space substantially simplifies the theoretical analysis of Brownian dynamics. In particular, diffusion in heterogeneous porous media was

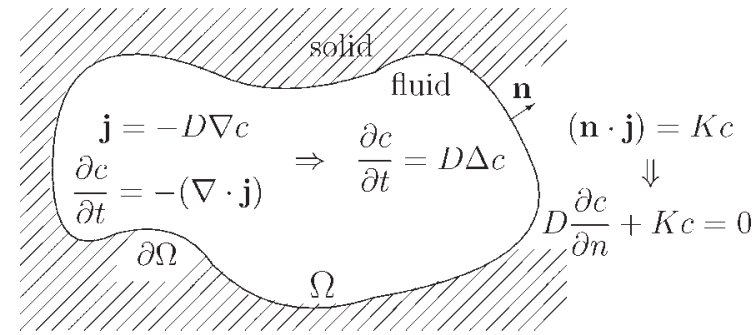

Figure 1 A solid matrix (shaded region) delimits the confining domain $\Omega$ which is filled with a liquid or a gas containing spin-bearing particles (white region). In the bulk, the flux density $\mathbf{j}(\mathbf{r}, t)$, determining the evolution of the density $c(\mathbf{r}, t)$, is proportional to its gradient (Fick's law), yielding the diffusion equation. In turn, the flux of particles from the bulk toward the boundary $\partial \Omega,(\mathbf{n} \cdot \mathbf{j})$, is equal to the flux of particles through the boundary, $K c$, yielding the Robin boundary condition.

often investigated by effective approaches based on Eq. [6]. Though successful in some aspects, these approaches lack accurate accounting for geometrical features of the confining medium.

\section{Boundary Condition}

When the motion of diffusing particles is restricted by a geometrical confinement (Fig. 1), the microscopic interaction between the particles and the boundary should be taken into account. These interactions may have different physicochemical or biological mechanisms. For instance, paramagnetic impurities dispersed on the boundary cause surface relaxation in NMR experiments; cellular membranes allow for a permeable transport through the boundary; chemical reaction may change the diffusive or magnetic properties of the particle, etc. A realistic description of these processes at the microscopic level is a challenging problem, demanding for example accurate molecular dynamics simulations near the interface, or quantum mechanics calculations. At the time scale of the macroscopic transport process, however, the contact with the interface is very rapid so that the precise description of the interaction is often irrelevant. In analogy with the diffusion coefficient $D$, effectively representing the bulk dynamics, the interactions on the boundary can macroscopically be described by a surface transport coefficient $K(11$, 12). In biology, this is the permeability characterizing the rate of transfer across a permeable membrane. In heterogeneous catalysis, $K$ is the reactivity of a catalyst, that is the rate at which diffusing species are chemically transformed into other species after 
hitting the boundary. In NMR, $K$ is the surface relaxivity determining the rate at which the nuclei lose their transverse magnetization in the vicinity of the boundary. In the two latter cases, the transformed or relaxed species still remain in the confinement but they do not participate in the transport process any more (e.g., they do not contribute to the formation of the spin-echo signal).

At macroscopic level, the boundary condition is a kind of mass conservation law. The flux of particles from the bulk toward the boundary is obtained by projecting the flux density $\mathbf{j}$ from Eq. [1] onto the unit normal vector $\mathbf{n}(\mathbf{r})$ at the boundary. On the other hand, the flux through the boundary (or the flux of transformed or relaxed species) is the product of the density of particles at the boundary and the surface transport coefficient $K$. Equating these two fluxes yields the Robin (also known as Fourier, mixed, relaxing, radiation, or third) boundary condition for the density at the boundary $\partial \Omega$ :

$$
D \frac{\partial}{\partial n} c(\mathbf{r}, t)+K c(\mathbf{r}, t)=0 \quad(\mathbf{r} \in \partial \Omega)
$$

where $\partial / \partial n$ is the normal derivative [the projection of the gradient operator $\nabla$ onto the unit vector $\mathbf{n}(\mathbf{r})$ ], pointing towards the exterior of the region (Fig. 1). In other words, the normal derivative shows the variation of a function in the spatial direction which is orthogonal to the boundary. For instance, when the boundary is a sphere, $\partial / \partial n$ reduces to $\partial / \partial r$ in radial coordinate. When the surface transport coefficient $K$ is zero (no flux through the boundary), one retrieves the Neumann boundary condition: $\partial c(\mathbf{r}, t) / \partial n=0$ at $\mathbf{r} \in \partial \Omega$. The opposite limit of infinite $K$ (no resistance to the transfer through the boundary) corresponds to the Dirichlet boundary condition: $c(\mathbf{r}, t)=0$ at $\mathbf{r} \in \partial \Omega$. The intermediate Robin boundary condition is then a linear combination of these two extreme cases, which are "weighted" by bulk and surface transport coefficients $D$ and $K$.

Similar boundary condition can be written for the propagator

$$
D \frac{\partial}{\partial n} G_{t}\left(\mathbf{r}_{0}, \mathbf{r}\right)+K G_{t}\left(\mathbf{r}_{0}, \mathbf{r}\right)=0 \quad(\mathbf{r} \in \partial \Omega) .
$$

Because the propagator represents a "family" of functions parametrized by the starting point $\mathbf{r}_{0}$, the above boundary condition should be satisfied for any $\mathbf{r}_{0}$. It is important to stress that the presence of boundary substantially modifies the solution of the diffusion equation [2]. In particular, the Gaussian propagator from Eq. [6] for the whole space does not satisfy the above boundary condition for a bounded domain. In other words, each confining domain has its own diffusive propagator which also depends on the transport parameters $D$ and $K$.

The surface relaxivity $K$ is assumed to be uniform over the boundary. This assumption is very common in the literature, by mainly three reasons:

- the real distribution of the surface relaxivity is unknown and practically inaccessible in most experiments;

- the implementation of a given distribution was considered as a significant additional complication of the problem;

- for long enough observation time, diffusion is expected to average out such inhomogeneities.

In Section "Relaxation Mechanisms", we shall explain how a given distribution of the surface relaxivity can be easily implemented in the matrix technique.

\section{Magnetic Field Encoding}

An observation of the dynamics requires a kind of "marking" or "labeling" of the traveling particles in order to trace their displacements in the confinement. Magnetic field is a superb experimental tool for encoding the motion of spin-bearing particles (13). Once the magnetization is flipped into the transverse plane by a $90^{\circ}$ radio-frequency (rf) pulse, the spins at position $\mathbf{r}$ precess with the Larmor frequency $\gamma B(\mathbf{r}, t)$ which is proportional to the magnetic field $B(\mathbf{r}, t), \gamma$ being the gyromagnetic ratio (a fundamental constant of the nucleus). The use of a spatially inhomogeneous magnetic field allows one to distinguish different points or regions in the bulk. This mechanism has been broadly applied in experiments to monitor the dynamics and to access the geometry of a confining medium.

Bloch-Torrey Equation. In 1956, Torrey modified the Bloch equation describing the evolution of the complex-valued transverse magnetization $\mathrm{m}(\mathbf{r}, t)$ to include the effect of diffusion (14):

$$
\begin{aligned}
& \frac{\partial}{\partial t} \mathrm{~m}(\mathbf{r}, t)=D \Delta \mathrm{m}(\mathbf{r}, t)-i \gamma B(\mathbf{r}, t) \mathrm{m}(\mathbf{r}, t) . \\
& \text { evolution : due to diffusion due to encoding }
\end{aligned}
$$

On the other hand, this Bloch-Torrey equation can also be considered as a diffusion equation, to which the effect of magnetic field encoding has been added. This equation states that the evolution of 
magnetization during a short time $\tau$ is caused by two independent mechanisms:

- diffusive migration of the spin-bearing particles (from $\mathbf{r}$ to neighboring points), represented by the Laplace operator and characterized by the diffusion coefficient $D$;

- magnetic field encoding, when the spins at $\mathbf{r}$ acquire the phase shift $\gamma B(\mathbf{r}, t) \tau$ resulting from their precession.

The macroscopic signal at time $t$ is formed by the whole ensemble of the spins:

$$
E=\int d \mathbf{r} \mathrm{m}(\mathbf{r}, t) \tilde{\rho}(\mathbf{r}),
$$

where $\tilde{\rho}(\mathbf{r})$ is a sampling or pickup function of the measuring coil or antenna. Usually one tries to design antenna to get $\tilde{\rho}(\mathbf{r})$ as uniform as possible.

Applied Magnetic Field. In most practical situations, the encoding term $B(\mathbf{r}, t)$ is a superposition $B_{0}+f(t)(\mathbf{g} \cdot \mathbf{r})$ of a static magnetic field $B_{0}$ and a linear magnetic field gradient $\mathbf{g}$, whose dependence on time is represented through a dimensionless temporal profile $f(t)$. The constant term $-i \gamma B_{0} \mathrm{~m}(\mathbf{r}, t)$ in Eq. [8] can then be dropped out because it leads to an explicit factor $\exp \left[-i \gamma B_{0} t\right]$. In turn, the inhomogeneous time-dependent gradient is responsible for highly nontrivial behavior, with crucial consequences both from physical and mathematical points of view.

The form of the temporal profile can be easily varied in modern MR scanners. The simplest choice $f(t)=1$ corresponds to a free induction decay (FID) in a constant gradient. In turn, two identical gradient pulses of opposite polarities [Fig. 2(a)] can be applied to form a gradient echo. If the nuclei were immobile, their dephasing by the first gradient pulse would be fully compensated by the second gradient pulse. When the nuclei diffuse, the rephasing is not complete, and the gradient-echo amplitude attenuation can be used to characterize restricted diffusion.

The dephasing/rephasing mechanism is similar to how a droplet of a colored liquid spreads in a laminar flow and then (partially) recovers its shape when the flow is reversed. In fact, each molecule moves along a streamline with a constant velocity which depends on its initial position. If the molecules remained on the initial streamlines, they would return to their initial positions when the flow is reversed. In practice, the recovered droplet shape is "blurred" or even fully destroyed by diffusion in the transverse plane (perpendicular to the flow direction) which allows the molecules to jump randomly between streamlines.
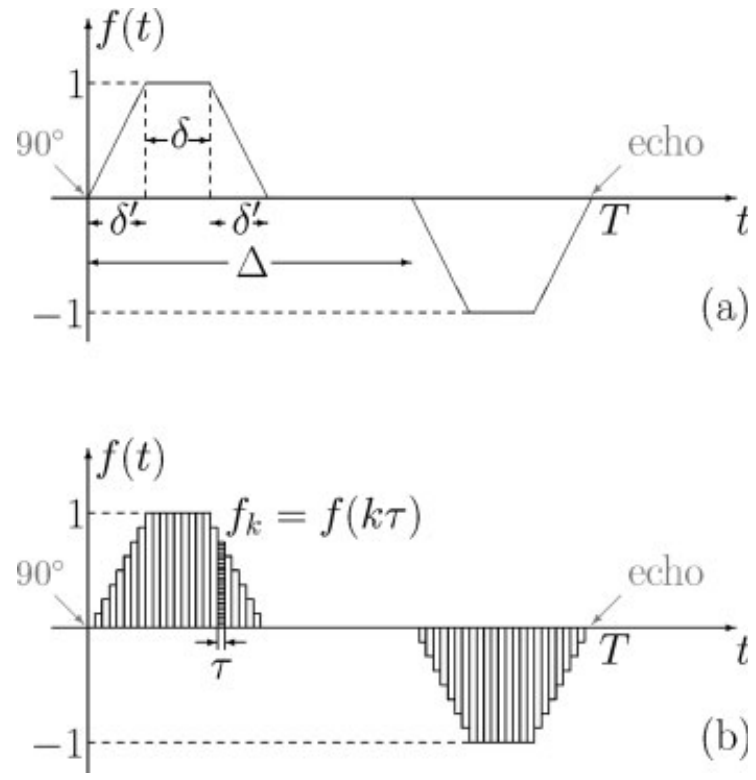

Figure 2 (a) Typical form of the temporal profile $f(t)$. After the exciting $90^{\circ} \mathrm{rf}$ pulse, the nuclei are dephased and rephased by two gradient pulses of opposite polarities. Their trapezoidal shape is characterized by the ramp time $\delta^{\prime}$, plateau duration $\delta$, and diffusion time $\Delta$. A gradient echo is formed at the echo time $T=\Delta+\delta+2 \delta^{\prime}$. The same profile $f(t)$ can also describe a spin-echo formation, when the opposite polarity of the second gradient pulse effectively accounts for the magnetization inversion by the $180^{\circ} \mathrm{rf}$ pulse. (b) A piecewise constant approximation of the above temporal profile. The choice of the time step $\tau$ is a compromise between the accuracy of the piecewise constant approximation (smaller $\tau$, better the accuracy) and the computational time (smaller $\tau$, longer the computation).

For spin-echo experiments, the gradient pulses have the same polarity, while the (partial) rephasing is realized by inverting the magnetization with a refocusing $180^{\circ}$ rf pulse (13). Although the physical mechanism is different, the inversion by the $180^{\circ} \mathrm{rf}$ pulse can be effectively taken into account by the same temporal profile $f(t)$ with opposite gradient polarities. In other words, we do not distinguish gradient and spin echoes when studying restricted diffusion. Similarly, the Carr-Purcell-Meiboom-Gill (CPMG) and stimulated spin-echo sequences can be handled with an appropriate choice of $f(t)(15)$.

Boundary Condition. Although Eq. [8] was originally deduced for the whole space, it can be readily applied to describe restricted diffusion by specifying the boundary condition. 
As in Section "Boundary Condition", the Robin boundary condition is often imposed

$$
D \frac{\partial}{\partial n} \mathrm{~m}(\mathbf{r}, t)+K \mathbf{m}(\mathbf{r}, t)=0 .
$$

While Eq. [8] governs the dynamics of spins in the bulk, the boundary condition tells what happens when the spin-bearing particle encounters the interface.

\section{Length Scales and Dimensionless Parameters.} The aforementioned mechanisms (diffusion and encoding) for the magnetization evolution, diffusion and encoding, provide us with two important length scales: the diffusion length $\sqrt{D T}$ showing how far the nuclei diffuse on average until the echo time $T$; and the gradient length $(\gamma g T)^{-1}$, over which the magnetic field gradient $g$ yields a phase spread of the order of $2 \pi$. When there is no boundary (unrestricted diffusion in the whole space), these are the only two scales of the problem. Because the signal attenuation is a dimensionless factor, the diffusion and gradient lengths necessarily enter as a ratio $\sqrt{D T} \gamma g T$ which is the only relevant parameter. Of course, this ratio can be multiplied by a number or, in general, one can use any nontrivial function of it as a new dimensionless variable. In particular, the square of this ratio over $D$, $\gamma^{2} g^{2} T^{3}$, is known, up to a numerical factor, as $b$ value. The dimensionless combination $D \gamma^{2} g^{2} T^{3}$ determines the way in which different physical parameters should be rescaled to get the same signal. In other words, the signal attenuation that would be deduced from an appropriate mathematical framework must be a function of this single parameter. In turn, finding the particular form of this function would require further analysis. For instance, the spinecho signal in a constant gradient is $(16,17)$ :

$$
E \propto \exp \left[-D \gamma^{2} g^{2} T^{3} / 12\right]
$$

For restricted diffusion, a new dimensional parameter, the size $L$ of the confining medium, should be introduced, leading to two dimensionless combinations which we denote as

$$
p=D T / L^{2} \quad \text { and } \quad q=\gamma g L T .
$$

It is worth noting that this choice of the dimensionless variables is conventional. For instance, one could alternatively take the previous combination $D \gamma^{2} g^{2} T^{3}$ instead of $p$ or $q$ or, in general, any two independent nontrivial functions of $p$ and $q$. Once a convenient pair of the two dimensionless variables is chosen, it should be kept through all the computation. We bring the attention to the distinction between the above dimensionless gradient intensity $q$ and the dimensional combination $\gamma g \delta / 2 \pi$ from the classical $q$-analysis with narrow gradient pulses of duration $\delta$. The former $q$ value is defined for any temporal profile and can thus be considered as an extension of the latter (classical) $q$ value.

Even at a qualitative level, it is clear that the existence of two independent parameters for restricted diffusion drastically complicates the problem, allowing for various regimes of the signal attenuation. In particular, the classical form [11] of the signal does not necessarily hold, as illustrated later.

Another attenuation mechanism would introduce a new length scale and a new dimensionless variable. For instance, surface relaxation which can be introduced via Eq. [10] brings the relaxation length $D / K$ which is the distance a particle should travel near the boundary before surface relaxation effects reduce its expected magnetization. The relaxation length is also called "unscreened perimeter length" and it plays an important role in diffusive transport phenomena (10, 11, 18-24). The third dimensionless variable

$$
h=\frac{K L}{D}
$$

further extends the diversity and complexity of diffusive NMR phenomena.

Conventions and Ambiguities. Although the above dimensional analysis correctly illustrates the concepts, it tells only half of the story. In practice, other "ingredients" (beside the physical parameters) have to be included to get a quantitative description. These are: the temporal and spatial profiles of the applied magnetic field; and the geometry of the confining medium. For sake of simplicity, we shall consider only a linear magnetic field gradient [other spatial profiles can also be implemented, see (1)].

The signal attenuation depends on the choice of the temporal profile $f(t)$. In a typical gradient-echo or spin-echo experiment, the gradient pulses have a trapezoidal shape which is characterized by the ramp time $\delta^{\prime}$, plateau duration $\delta$, and diffusion time $\Delta$ [Fig. 2(a)]. The following question naturally arises: which time characteristic of the temporal profile should be used as the time scale $T$ ? Is it the plateau duration $\delta$ or some effective duration of one dephasing pulse? Or the diffusion time $\Delta$ ? Or the accumulated duration of both pulses when the gradient is on? Or the whole duration of the temporal profile, including the wait- 
ing time between two pulses? Or the echo time? There may be various speculations about which time characteristics would be physically more relevant, but they are pointless. In fact, the signal attenuation is determined by both the whole temporal profile $f(t)$ and its timing characteristics, and not by our particular preference for the time scale. Once the time scale is conveniently chosen, it is important to use it coherently throughout the whole computation. In what follows, we always take $T$ to be the echo time.

A similar freedom of choice (and a possible ambiguity) occurs for the characteristic length scale $L$ of the confining domain. For diffusion between two parallel planes, choosing for $L$ the separation width is rather natural. However, should $L$ be the diameter or the radius for a sphere? The same question becomes challenging for heterogeneous porous media when multiple length scales are present: Should $L$ be the smallest pore scale or the largest sample size? Should it be a kind of an average length (such as, for example, the ratio between the volume and the surface area)? Again, there is no definite answer to this question of conventional matter. The restricted diffusion and the consequent signal attenuation depend on the whole geometry of the confining medium, and the characteristic length $L$ is only used to get dimensionless variables. As shown below, the signal attenuation is determined by the matrices $\Lambda, \mathcal{B}$, and $\tilde{\mathcal{B}}^{s}$ representing the geometry. These matrices have to be calculated, analytically or numerically, for a confining medium of a fixed (unit) size. A dilatation of this medium is then represented by the length scale $L$. In practice, the choice of the length $L$ is therefore set by the formulas used for the governing matrices. For instance, the formulas in Table 1 are derived under the convention that $L$ is the separation width for a slab and the radius for a cylinder and a sphere.

To summarize this discussion, we stress again that the choice of the dimensionless combinations $p, q$, and $h$ of physical parameters is conventional, but it should be coherent throughout the whole computation. In addition to physical parameters, the confining geometry and the applied magnetic field come into the description. In particular, two measurements with the same values of $p, q$, and $h$ but in different geometries would lead to different results.

\section{Notion of Eigenfunction}

The notion of eigenfunction is as simple as fundamental. In the next subsections, we first illustrate this notion for $2 \times 2$ matrices and then focus on the Laplace operator.
Table 1 Mathematical Basis for Computing the Governing Matrices $\Lambda, \mathcal{B}$, and $\widetilde{\mathcal{B}}^{s}$ for Three Confining Domains: Interval, Disk, and Sphere [More General Formulas for Robin Boundary Condition Are Given in (1)]

\begin{tabular}{l} 
1D: Interval/Slab, \\
$\Omega=\{x \in \mathbb{R}: 0<x<1\} \quad(V=1)$ \\
\hline $\sin \left(\alpha_{m}\right)=0 \Rightarrow \alpha_{m}=\pi m$ \\
$\lambda_{m}=\pi^{2} m^{2} \quad \Lambda_{m, m^{\prime}}=\delta_{m, m^{\prime}} \lambda_{m}$ \\
$u_{m}(x)=\varepsilon_{m} \cos (\pi m x)$ \\
$\varepsilon_{m}=\left(2-\delta_{m, 0}\right)^{1 / 2}$ \\
$\tilde{\mathcal{B}}_{m, m^{\prime}}^{s}=\varepsilon_{m} \varepsilon_{m^{\prime}}\left(1+(-1)^{m+m^{\prime}}\right)$ \\
$\mathcal{B}_{m, m^{\prime}}=\left((-1)^{m+m^{\prime}}-1\right) \varepsilon_{m} \varepsilon_{m^{\prime}} \frac{\lambda_{m}+\lambda_{m^{\prime}}}{\left(\lambda_{m}-\lambda_{m^{\prime}}\right)^{2}}, \quad \mathcal{B}_{m, m}=\frac{1}{2}$ \\
\hline
\end{tabular}

2D: Disk/Cylinder, $\Omega=\left\{\mathbf{r} \in \mathbb{R}^{2}:|\mathbf{r}|<1\right\} \quad(V=\pi)$

$J_{n}^{\prime}\left(\alpha_{n k}\right)=0$

$\lambda_{n k}=\alpha_{n k}^{2} \quad \Lambda_{n k, n^{\prime} k^{\prime}}=\delta_{n, n^{\prime}} \delta_{k, k^{\prime}} \lambda_{n k}$

$u_{n k}(r, \varphi)=\frac{\varepsilon_{n}}{\sqrt{\pi}} \frac{\beta_{n k}}{J_{n}\left(\alpha_{n k}\right)} J_{n}\left(\alpha_{n k} r\right) \cos (n \varphi)$

$\beta_{n k}=\left(\frac{\lambda_{n k}}{\lambda_{n k}-n^{2}}\right)^{1 / 2} \quad \beta_{00}=1$

$\tilde{\mathcal{B}}_{n k, n^{\prime} k^{\prime}}^{s}=2 \delta_{n, n^{\prime}} \beta_{n k} \beta_{n^{\prime} k^{\prime}}$

$\mathcal{B}_{n k, n^{\prime} k^{\prime}}=\delta_{n, n^{\prime} \pm 1}\left(1+\delta_{n, 0}+\delta_{n^{\prime}, 0}\right)^{1 / 2}$

$$
\times \beta_{n k} \beta_{n^{\prime} k^{\prime}} \frac{\lambda_{n k}+\lambda_{n^{\prime} k^{\prime}}-2 n n^{\prime}}{\left(\lambda_{n k}-\lambda_{n^{\prime} k^{\prime}}\right)^{2}}
$$

3D: Sphere, $\Omega=\left\{\mathbf{r} \in \mathbb{R}^{3}:|\mathbf{r}|<1\right\} \quad(V=4 \pi / 3)$

$j_{n}^{\prime}\left(\alpha_{n k}\right)=0$

$\lambda_{n k}=\alpha_{n k}^{2} \quad \Lambda_{n k, n^{\prime} k^{\prime}}=\delta_{n, n^{\prime}} \delta_{k, k^{\prime}} \lambda_{n k}$

$u_{n k}(r, \theta)=\frac{\beta_{n k}}{\sqrt{2 \pi} j_{n}\left(\alpha_{n k}\right)} j_{n}\left(\alpha_{n k} r\right) P_{n}(\cos \theta)$

$\beta_{n k}=\left(\frac{(2 n+1) \lambda_{n k}}{\lambda_{n k}-n(n+1)}\right)^{1 / 2} \quad \beta_{00}=\sqrt{3 / 2}$

$\tilde{\mathcal{B}}_{n k, n^{\prime} k^{\prime}}^{s}=2 \delta_{n, n^{\prime}} \frac{\beta_{n k} \beta_{n^{\prime} k^{\prime}}}{2 n+1}$

$\mathcal{B}_{n k, n^{\prime} k^{\prime}}=\delta_{n, n^{\prime} \pm 1} \frac{\left(n+n^{\prime}+1\right)}{(2 n+1)\left(2 n^{\prime}+1\right)} \beta_{n k} \beta_{n^{\prime} k^{\prime}} \times$

$$
\frac{\lambda_{n k}+\lambda_{n^{\prime} k^{\prime}}-n\left(n^{\prime}+1\right)-n^{\prime}(n+1)+1}{\left(\lambda_{n k}-\lambda_{n^{\prime} k^{\prime}}\right)^{2}}
$$

For each domain, we specify line by line: the equation for $\alpha_{m}$ (or $\alpha_{n k}$ ), the eigenvalues, the eigenfunctions, the normalization constants, and the matrices $\tilde{\mathcal{B}}^{s}$ and $\mathcal{B}$.

Illustration with $2 \times 2$ Matrices. We start by considering a vector $v=\left(\begin{array}{l}x_{1} \\ x_{2}\end{array}\right)$ in the plane and its lin- 
ear transformations by $2 \times 2$ matrices. For instance, the application of the matrix $\left(\begin{array}{cc}2 & 0 \\ 0 & 1 / 2\end{array}\right)$ to $v$ extends the first coordinate and shrinks the second one by factor 2. This geometric interpretation in terms of dilatations is clear since the diagonal structure of the matrix yields a separate action on both coordinates. When the matrix is not diagonal, its action is less obvious because the coordinates will be mixed. The spectral analysis is intended to get a clearer interpretation by diagonalizing the matrix. For example, what is a "geometric action" of the matrix $A=\left(\begin{array}{ll}3 / 4 & 1 / 4 \\ 1 / 4 & 3 / 4\end{array}\right)$ ? Can this action be interpreted in terms of dilatations, at least for some particular vectors? In other words, do exist vectors which are invariant under its action, up to a multiplicative factor? It is easy to check that the vectors

$$
u_{1}=\frac{1}{\sqrt{2}}\left(\begin{array}{l}
1 \\
1
\end{array}\right) \quad \text { and } \quad u_{2}=\frac{1}{\sqrt{2}}\left(\begin{array}{c}
1 \\
-1
\end{array}\right)
$$

are indeed invariant, and therefore called eigenvectors (also named as eigenfunctions, eigenstates, or eigenmodes) of the matrix $A: A u_{1}=u_{1}$ and $A u_{2}=(1 / 2) u_{2}$. These vectors are defined uniquely, up to normalization constants. The associated multiplicative factors $\lambda_{1}=1$ and $\lambda_{2}=1 / 2$ are called eigenvalues. Since any vector $v$ can be decomposed in a linear combination of the orthogonal vectors $u_{1}$ and $u_{2}$, the action of the matrix $A$ to $v$ is

$$
A v=\left(u_{1} \cdot v\right) \lambda_{1} u_{1}+\left(u_{2} \cdot v\right) \lambda_{2} u_{2}
$$

where the scalar products $\left(u_{i} \cdot v\right)$ are projections of $v$ onto $u_{1}$ and $u_{2}$. The fundamental role of the eigenvectors $u_{1}$ and $u_{2}$ is to provide a natural basis, in which the action of the matrix $A$ is reduced to dilatations (multiplication by a number).

The importance of such a spectral decomposition is far beyond the provided geometric interpretation. Since the action of a matrix in its own eigenbasis is just dilatations, its $k$-th power acts as dilatations as well:

$$
A^{k} v=\left(u_{1} \cdot v\right) \lambda_{1}^{k} u_{1}+\left(u_{2} \cdot v\right) \lambda_{2}^{k} u_{2} .
$$

This is a way to build a general matrix analysis. For instance, for a given function $F(x)$, one can define the matrix $F(A)$ by its action on a vector $v$ :

$$
F(A) v=\left(u_{1} \cdot v\right) F\left(\lambda_{1}\right) u_{1}+\left(u_{2} \cdot v\right) F\left(\lambda_{2}\right) u_{2} .
$$

We shall use this definition in what follows.

The Laplace Operator. The above spectral analysis is not restricted to the two-dimensional case. The very same concepts can be applied to finite-dimen- sional matrices or even infinite-dimensional linear operators acting on functions (instead of vectors) from some functional spaces (instead of the plane). This is the core of a mathematical formulation of quantum mechanics (25). The analysis is technically more delicate in infinite-dimensional spaces because convergence issues have to be addressed. Once again, the eigenfunctions, which remain invariant up to a multiplicative factor under the action of a linear operator, form a natural basis in the functional space. In its own eigenbasis, the linear operator acts by extending or shrinking the "directions" determined by the eigenfunctions. So, if a given function is decomposed in a linear combination of the eigenfunctions of a linear operator, this operator can be applied individually to each term of the sum, yielding another linear combination with easily computable coefficients. But does any linear operator possess the eigenfunctions? Are they complete so that any function can be decomposed? In general, the answers are no. Fortunately, the Laplace operator in a bounded domain with Dirichlet, Neumann, or Robin boundary condition has a complete set of eigenfunctions. The way how the Laplace operator acts is therefore fully represented through its eigenfunctions and eigenvalues, whatever the complexity of this action. In other words, finding the eigenfunctions and eigenvalues is equivalent to knowing the Laplace operator. It is thus not surprising that the computation of the eigenbasis is a difficult task, but once this step is accomplished, any property related to the operator can be investigated.

The Laplace operator is probably the most studied operator as being a part of many differential equations describing various phenomena: diffusion and heat transfer, wave propagation, free particle in quantum mechanics, to name a few. The eigenfunctions $u_{m}(\mathbf{r})$ and eigenvalues $\lambda_{m}$ are defined as

$$
\begin{array}{rlrl}
\Delta u_{m}(\mathbf{r})+\lambda_{m} u_{m}(\mathbf{r}) & =0 & & (\mathbf{r} \in \Omega), \\
D \frac{\partial}{\partial n} u_{m}(\mathbf{r})+K u_{m}(\mathbf{r}) & =0 & (\mathbf{r} \in \partial \Omega),
\end{array}
$$

with an integer index $m=0,1,2, \ldots$. Depending on the application field (e.g., acoustics, quantum mechanics, etc.), the interpretation of Laplacian eigenfunctions is different.

Example: One-Dimensional Case. Let us consider the unit interval: $\Omega=(0,1)$. In this one-dimensional case, the Laplace operator is simply the second-order spatial derivative, $\Delta=\partial^{2} / \partial x^{2}$. Evidently, $u_{m}(x)=$ $\sin (\pi m x)$ is an eigenfunction of this operator,

$$
-\frac{\partial^{2}}{\partial x^{2}} u_{m}(x)=\pi^{2} m^{2} u_{m}(x)
$$



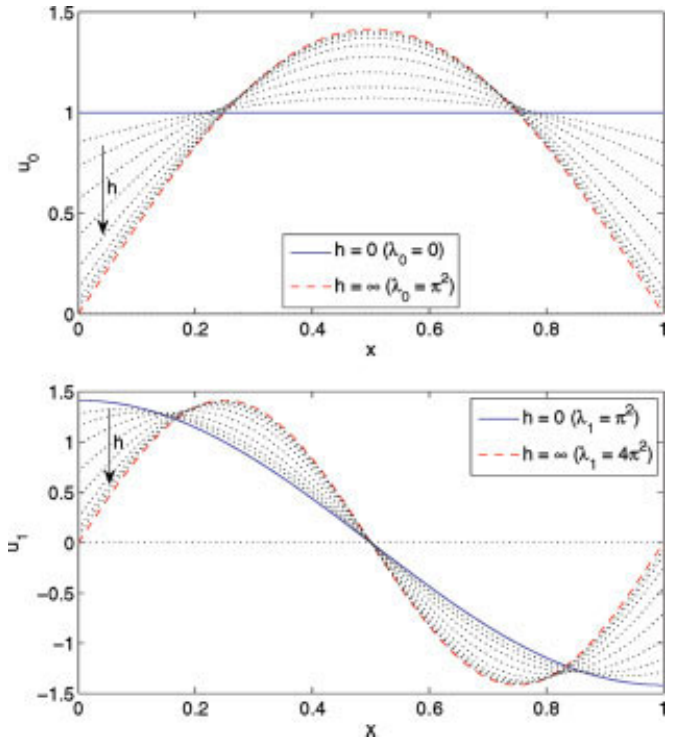

Figure 3 Example of two first eigenfunctions of the Laplace operator on the unit interval. The dimensionless surface relaxivity $h$ varies from 0 (Neumann boundary condition, solid blue line) to infinity (Dirichlet boundary condition, dashes red line). The successive intermediate curves correspond to $h$ taking values $1,2,4,8,16,32$, 64, and 128. [Color figure can be viewed in the online issue, which is available at www.interscience.wiley.com.]

satisfying the Dirichlet boundary condition: $u_{m}(0)=u_{m}(1)=0$. Note that $\tilde{u}_{m}(x)=\cos (\pi m x)$ is also an eigenfunction of the Laplace operator, but with the Neumann boundary condition: $\tilde{u}_{m}^{\prime}(0)=\tilde{u}_{m}^{\prime}(1)=0$, the prime denoting the spatial derivative (Fig. 3). In both cases, the corresponding eigenvalue $\lambda_{m}$ is $\pi^{2} m^{2}$.

In acoustics, the functions $\{\sin (\pi m x)\}$ are known as vibration modes or stationary waves of a string of length 1 with fixed end points. The square root of the eigenvalue, $\sqrt{\lambda_{m}}=\pi m$, is called the wavenumber (or spatial frequency) giving the number of half-periods, $\pi$, of the wave. Furthermore, Fourier analysis suggests to decompose a given initial profile of the string into a linear combination of these stationary waves in order to investigate its vibration. The very same concept is applicable for any shape of vibrating domain, when the Fourier harmonics are replaced by the related Laplacian eigenfunctions.

In quantum mechanics, $\{\sqrt{2} \sin (\pi m x)\}$ are the wave functions of a particle restricted to the unit interval by infinite potentials at $x \leq 0$ and $x \geq 1$. When the particle is at state $m, 2|\sin (\pi m x)|^{2}$ can be interpreted as the probability to find this particle at point $x$, while the eigenvalue $\lambda_{m}$ is proportional to the energy of this state. This interpretation remains true for any confining domain $\Omega$, when $\left\{u_{m}(\mathbf{r})\right\}$ are the Laplacian eigenfunctions in this domain.

General Properties. The self-adjointness of the Laplace operator (see Ref. (26) for definition and details) implies that the eigenvalues $\lambda_{m}$ are real, and the eigenfunction $u_{m}(\mathbf{r})$ are orthogonal:

$$
\int_{\Omega} d \mathbf{r} u_{m}(\mathbf{r}) u_{m^{\prime}}^{*}(\mathbf{r})=\delta_{m, m^{\prime}}
$$

where the asterisk denotes complex conjugate (note that throughout this paper, we deal with real-valued eigenfunctions, so that the asterisk can be omitted). The above equation also explicitly fixes the normalization and the dimensional units: the eigenfunctions are measured in meter ${ }^{-d / 2}$. We recall that the eigenvalues are measured in meter ${ }^{-2}$, independently of the dimension $d$.

The possibility to decompose a given function into a linear combination of the eigenfunctions is expressed through the completeness relation

$$
\delta\left(\mathbf{r}-\mathbf{r}^{\prime}\right)=\sum_{m} u_{m}(\mathbf{r}) u_{m}^{*}\left(\mathbf{r}^{\prime}\right)
$$

In fact, multiplying this relation by a function $f\left(\mathbf{r}^{\prime}\right)$ and integrating it over $\mathbf{r}^{\prime} \in \Omega$ yield the desired representation:

$$
f(\mathbf{r})=\sum_{m} u_{m}(\mathbf{r}) \underbrace{\int_{\Omega} d \mathbf{r}^{\prime} u_{m}^{*}\left(\mathbf{r}^{\prime}\right) f\left(\mathbf{r}^{\prime}\right)}_{c_{m}},
$$

the integrals providing the coefficients $c_{m}$. This spectral decomposition is an extension of classical Fourier series to the functions which are defined on an arbitrary bounded domain $\Omega$. Moreover, the diffusive propagator has a similar representation

$$
G_{t}\left(\mathbf{r}, \mathbf{r}^{\prime}\right)=\sum_{m} u_{m}^{*}(\mathbf{r}) u_{m}\left(\mathbf{r}^{\prime}\right) e^{-D \lambda_{m} t} .
$$

One can easily check that this sum satisfies the diffusion equation [2], while the initial condition [4] at $t=0$ is guaranteed by the completeness relation [16].

It is clear that the eigenfunctions and eigenvalues explicitly determine the diffusive propagator. The opposite is also true: the propagator can be formally used to determine the eigenfunctions and eigenvalues. In fact, multiplying Eq. [18] by $u_{m^{\prime}}(\mathbf{r})$ and integrating over $\mathbf{r} \in \Omega$ yield the following integral 


$$
\begin{aligned}
& G_{t}\left(x, x^{\prime}\right)= \\
& e^{-D \lambda_{0} t} u_{0}(x) u_{0}\left(x^{\prime}\right)
\end{aligned}
$$

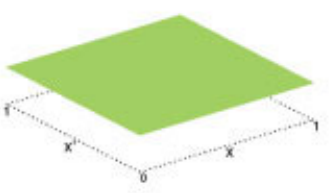

$+$

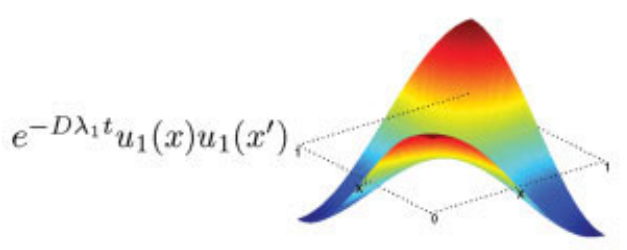

$$
+
$$
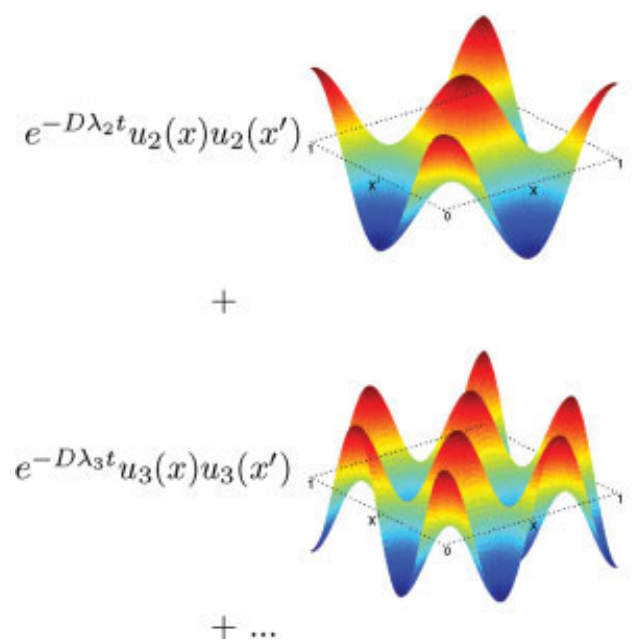
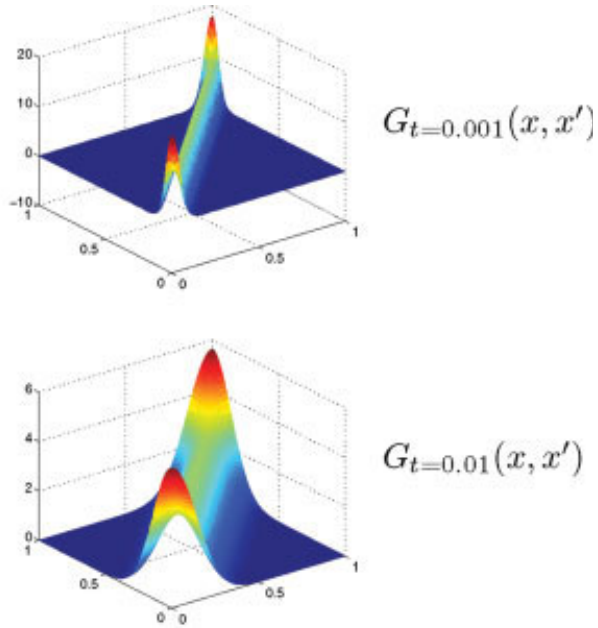

$G_{t=0.01}\left(x, x^{\prime}\right)$

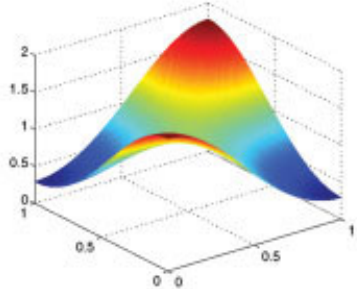

$G_{t=0.1}\left(x, x^{\prime}\right)$

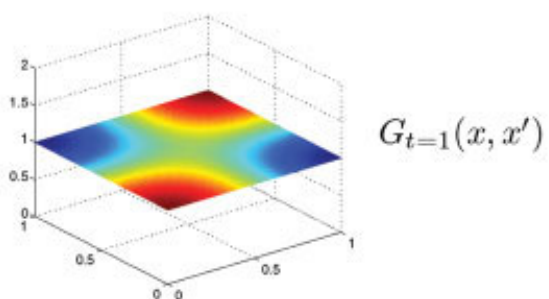

Figure 4 Illustration for the spectral decomposition of the diffusive propagator on the unit interval. On the left, the first four terms of the spectral decomposition: the contribution of each product $u_{m}(x) u_{m}\left(x^{\prime}\right)$ of the eigenfunctions (here, cosine functions) is "weighted" by the time-dependent factor $e^{-D \lambda_{m} t}$ (here, all the products are shown with the unit weights corresponding to $t=0$ ). For small $t$ (the first two plots on the right), the oscillating character and large number of contributing eigenfunctions make the propagator to concentrate near the diagonal $x=x^{\prime}$ (in agreement with the limit $G_{t}\left(x, x^{\prime}\right) \rightarrow \delta\left(x-x^{\prime}\right)$ as $t \rightarrow 0$ ). For larger $t$, the highly oscillating eigenmodes become more and more attenuated by their time-dependent weights. For instance, the third plot on the right shows $G_{t}\left(x, x^{\prime}\right)$ for $t=0.1$ for which only the eigenmodes $m=0$ and $m=1$ still significantly contribute. For $t=1$, the only contribution of the constant ground state $m=0$ with $\lambda_{0}=0$ remains significant. [Color figure can be viewed in the online issue, which is available at www.interscience.wiley.com.]

equation for the eigenfunctions:

$$
\int_{\Omega} d \mathbf{r} G_{t}\left(\mathbf{r}, \mathbf{r}^{\prime}\right) u_{m^{\prime}}(\mathbf{r})=e^{-D \lambda_{m^{\prime}} t} u_{m^{\prime}}\left(\mathbf{r}^{\prime}\right) .
$$

This is another confirmation that the diffusive propagator contains all available information about diffusive motion. In fact, the same amount of infor- mation is "stored" differently in the eigenbasis and in the propagator, the latter mixing this information in a specific way (Fig. 4). Figuratively speaking, one can store numerous sheets in various appropriate boxes (eigenfunctions), or put them together in a large single folder (propagator).

It is worth noting that the eigenvalues can be expressed through the corresponding eigenfunctions 
by multiplying Eq. [14a] by $u_{m}^{*}(\mathbf{r})$ and integrating over $\Omega$

$$
\lambda_{m}=\underbrace{\int_{\Omega} d \mathbf{r}\left|\nabla u_{m}(\mathbf{r})\right|^{2}}_{\text {bulk "kinetic energy }}+\frac{K}{D} \underbrace{\int_{\partial \Omega} d \mathbf{r}\left|u_{m}(\mathbf{r})\right|^{2}}_{\text {surface "potential energy }}
$$

(here we used the Green formula to integrate by parts and Eq. [14b] to express the normal derivative of the eigenfunction). In particular, this relation shows that all eigenvalues are positive. In analogy with quantum mechanics or acoustics, these terms can be respectively interpreted as the bulk kinetic energy and the potential energy which is localized in the surface region (27).

\section{MATRIX FORMALISM}

As we already mentioned, Laplacian eigenfunctions are the proper "letters" in a mathematical language describing diffusive phenomena. It is difficult to trace back their first use for this purpose. Robertson applied a quantum-mechanical operator formalism to study restricted diffusion in a slab geometry between two parallel planes (28). This approach was reformulated and further extended by Neuman (29). The importance of the Laplacian eigenfunctions was also recognized by Brownstein and Tarr in their study of surface relaxation in a geometrical confinement (30).

The Robertson's approach can be considered as a prototype for the efficient numerical techniques that we describe in this Section. In fact, a numerical resolution of the Bloch-Torrey equation [8] in a given domain is computationally time consuming for intense gradients or long times. To overcome this problem, Caprihan et al. proposed an original numerical approach (31). The idea was to approximate a given temporal gradient profile $f(t)$ by a large sum of equidistant very narrow gradient pulses. The solution can then be obtained by a successive use of the narrowpulse approximations. This so-called "multiple propagator approach" was reformulated by Callaghan in an elegant matrix form (32). He showed that the macroscopic signal can be written within a matrix formalism involving the Laplace operator eigenbasis. In practice, the numerical problem of finding the signal attenuation under an arbitrary temporal gradient profile was reduced to symbolic manipulation with two matrices which depend on several physical parameters, and on the confining geometry. In further works, Callaghan and Codd studied restricted diffusion in a cylinder and a sphere and discussed the role of surface relaxation $(33,34)$. Another formulation based on random walks was given by Sukstanskii and Yablonskiy (35). Finally, Barzykin proposed an equivalent matrix formalism by considering a stepwise approximation of the temporal gradient profile $(36,37)$. In his approach, the two matrices determining the macroscopic signal depend solely on the confining geometry. These matrices have thus to be calculated only once for a chosen confining medium (e.g., a sphere), after that the computation of the signal is straightforward and rapid for any set of physical parameters. This was an important improvement of the above matrix techniques and a crucial simplification for numerical analysis. The numerical tool presented in this Section essentially relies on the Barzykin's stepwise gradient approximation.

The different matrix approaches outlined earlier were mainly intended for numerical computation of the macroscopic signal. Axelrod and Sen developed a systematic formalism for calculating the magnetization of spins diffusing in a bounded region in the presence of surface relaxation and general magnetic field inhomogeneity (38). This approach was reformulated, extended and used by the author as the appropriate mathematical language for reviewing vast literature on diffusive phenomena in NMR (1). In this Section, we shall use the notations and concepts following (1).

\section{Exact Solution for Time-Independent Magnetic Fields}

The imaginary term with an inhomogeneous magnetic field in the Bloch-Torrey equation [8] is a significant complication. In spite of this fact, many results can still be deduced by considering the magnetic field as a "perturbation" of the Laplace operator. We first investigate the case when the applied magnetic field is independent of time. The above equation in dimensionless units is

$$
\left(T \frac{\partial}{\partial t}-(\underbrace{D T / L^{2}}_{p}) L^{2} \Delta+i(\underbrace{\gamma g T L}_{q}) B(\mathbf{r})\right) \mathrm{m}(\mathbf{r}, t)=0,
$$

where $B(\mathbf{r})$ is the normalized dimensionless spatial profile of the applied magnetic field: $B(\mathbf{r})=(\mathbf{e} \cdot \mathbf{r}) / L$ for a linear gradient in spatial direction e. One can recognize the dimensionless parameters $p$ and $q$ in front of the diffusive and encoding terms, respectively. 
As in quantum mechanics, the eigenfunctions $u_{m}(\mathbf{r})$ of the "unperturbed Hamiltonian" (here, the Laplace operator) serve as a basis to decompose the solution of Eq. [19]:

$$
\mathrm{m}(\mathbf{r}, t)=\sum_{m^{\prime}} c_{m^{\prime}}(t) u_{m^{\prime}}(\mathbf{r})
$$

with unknown time-dependent coefficients $c_{m^{\prime}}(t)$. Substitution of this expansion in Eq. [19], multiplication by $u_{m}^{*}(\mathbf{r})$, and integration over $\Omega$ yield a set of ordinary differential equations on the coefficients $c_{m}(t)$

$$
T \frac{\partial c_{m}(t)}{\partial t}+\sum_{m^{\prime}}\left(p \Lambda_{m, m^{\prime}}+i q \mathcal{B}_{m, m^{\prime}}\right) c_{m^{\prime}}(t)=0
$$

where the infinite-dimensional matrices $\Lambda$ and $\mathcal{B}$ represent the "unperturbed Hamiltonian" (the Laplace operator) and the "perturbing interaction" (the magnetic field) in the eigenbasis of the Laplace operator:

$$
\begin{gathered}
\mathcal{B}_{m, m^{\prime}}=\int_{\Omega} d \mathbf{r} u_{m}^{*}(\mathbf{r}) B(\mathbf{r}) u_{m^{\prime}}(\mathbf{r}), \\
\Lambda_{m, m^{\prime}}=\delta_{m, m^{\prime}} \lambda_{m} L^{2}
\end{gathered}
$$

Thinking of $c_{m}(t)$ as components of an infinitedimensional vector $C(t)$ leads to a matrix first-order differential equation

$$
T \frac{d C(t)}{d t}=-(p \Lambda+i q \mathcal{B}) C(t)
$$

As for a scalar equation, the solution is the matrix exponential (see below):

$$
\sqrt{V} C(t)=U e^{-(p \Lambda+i q \mathcal{B}) t / T}
$$

where a supplementary factor $\sqrt{V}$ is put to compensate the dimensional unit, meter ${ }^{-d / 2}$, of the vector $C(t)$ ( $V$ being the volume of the domain). Here the matrix exponential $e^{-(p \Lambda+i q \mathcal{B}) t / T}$ acts on the left on the vector $U$ representing the initial density $\rho(\mathbf{r})$ in the basis of eigenfunctions $\left\{u_{m}(\mathbf{r})\right\}$ :

$$
U_{m}=V^{1 / 2} \int_{\Omega} d \mathbf{r} u_{m}^{*}(\mathbf{r}) \rho(\mathbf{r}) .
$$

The macroscopic signal is then obtained according to Eq. [9] by integrating the magnetization $\mathrm{m}(\mathbf{r}, t)$ over the whole confining domain $\Omega$ with a sampling or pickup function $\tilde{\rho}(\mathbf{r})$ of the measuring coil or antenna:

$$
\begin{aligned}
E & =\int_{\Omega} d \mathbf{r} \mathrm{m}(\mathbf{r}, t) \tilde{\rho}(\mathbf{r}) \\
& =\sum_{m} c_{m}(t) \underbrace{V^{-1 / 2} \int_{\Omega} d \mathbf{r} u_{m}(\mathbf{r}) \tilde{\rho}(\mathbf{r})}_{\tilde{U}_{m}} .
\end{aligned}
$$

The last sum can be interpreted as a scalar product between the vector $C(t)$ and the vector $\tilde{U}$ representing the pickup function $\tilde{\rho}(\mathbf{r})$ in the basis of the eigenfunctions $\left\{u_{m}(\mathbf{r})\right\}$ :

$$
\tilde{U}_{m}=V^{-1 / 2} \int_{\Omega} d \mathbf{r} u_{m}(\mathbf{r}) \tilde{\rho}(\mathbf{r}) .
$$

The macroscopic signal at time $t$ can thus be written in a compact matrix form of a scalar product:

$$
E=\left(U e^{-(p \Lambda+i q \mathcal{B}) t / T} \tilde{U}\right)
$$

It is worth recalling that here and throughout this paper, we use the convention for matrices to act on the left.

In summary, the Laplace operator and the magnetic-field term in the Bloch-Torrey equation [8] were respectively considered as an unperturbed (free) Hamiltonian and a perturbing interaction. From such a quantum-mechanical point of view, the matrix $\mathcal{B}$ corresponds to the representation of the perturbing interaction in the basis of the free Hamiltonian (in such a basis, the matrix $\Lambda$ is necessarily diagonal). The matrix $e^{-(p \Lambda+i q \mathcal{B}) t / T}$ can thus be thought of as a kind of evolution operator acting on the initial state $\rho(\mathbf{r})$ (represented by the vector $U$ ). The resulting density $\mathrm{m}(\mathbf{r}, t)$ is then weighted by the pickup or sampling function $\tilde{\rho}(\mathbf{r})$ (represented by vector $\tilde{U}$ ). It is worth noting that the matrices $\mathcal{B}$ and $\Lambda$ do not commute.

We bring the reader's attention to one important point. Although the above derivation was performed in the spirit of a quantum-mechanical perturbation theory, the result [29] is exact, no simplifying approximation has been involved. An approximation will come later when the infinite-dimensional matrices $\mathcal{B}$ and $\Lambda$ will be truncated to finite sizes for practical implementation. We shall discuss these issues in Sections "Numerical Implementation" and "Choice of Truncation size".

Note: The matrix exponential $e^{X}$ can be defined and calculated in several ways:

(1) One can use the Taylor series expansion of the exponential function, 


$$
e^{X}=\sum_{k=0}^{\infty} \frac{X^{k}}{k !}
$$

where a scalar is replaced by a matrix $X$. For finite-dimensional matrices, this series always converges. However, if the matrix $X$ is infinite-dimensional (as in our case), its powers $X^{k}$ may be infinite while the exponential matrix $e^{X}$ can still exist.

(2) Alternatively, the matrix exponential can be represented as the limit

$$
e^{X}=\lim _{\varepsilon \rightarrow 0}(I+\varepsilon X)^{1 / \varepsilon}
$$

where $I$ stands for the identity matrix. This relation is often used for computing the matrix exponentials in practice.

(3) When the matrix $X$ is diagonalizable, a spectral definition like Eq. [13] is often the most appropriate for theoretical and numerical purposes.

It is also worth noting that some classical properties of the exponential function are not applicable for matrices. In particular, the most usual property $e^{X+Y}=e^{X} e^{Y}$ does not hold, unless the two matrices
$X$ and $Y$ commute: $X Y=Y X$. For instance, the matrices

$$
X=\left(\begin{array}{ll}
1 & 0 \\
0 & 0
\end{array}\right) \quad \text { and } \quad Y=\left(\begin{array}{ll}
0 & 1 \\
0 & 0
\end{array}\right)
$$

do not commute, so that the three matrices

$$
\begin{aligned}
e^{X+Y} & =\left(\begin{array}{ll}
e & e \\
0 & 1
\end{array}\right) \quad e^{X} e^{Y}=\left(\begin{array}{cc}
e & e-1 \\
0 & 1
\end{array}\right) \\
e^{Y} e^{X} & =\left(\begin{array}{ll}
e & 1 \\
0 & 1
\end{array}\right)
\end{aligned}
$$

are distinct.

\section{Piecewise-Constant Temporal Profiles}

The compact matrix form [29] was derived for timeindependent magnetic fields that was crucial for solving the set of differential equations [21]. However, the same approach can be applied in a much more general situation when the temporal profile of the magnetic field is a piecewise constant function. As an example of practical importance, we consider Stejskal-Tanner bipolar gradient pulses of rectangular shape and duration $\delta$ (Fig. 5). The first gradient pulse starts at time $t=0$ and ceases at $t=\delta$, while the sec-

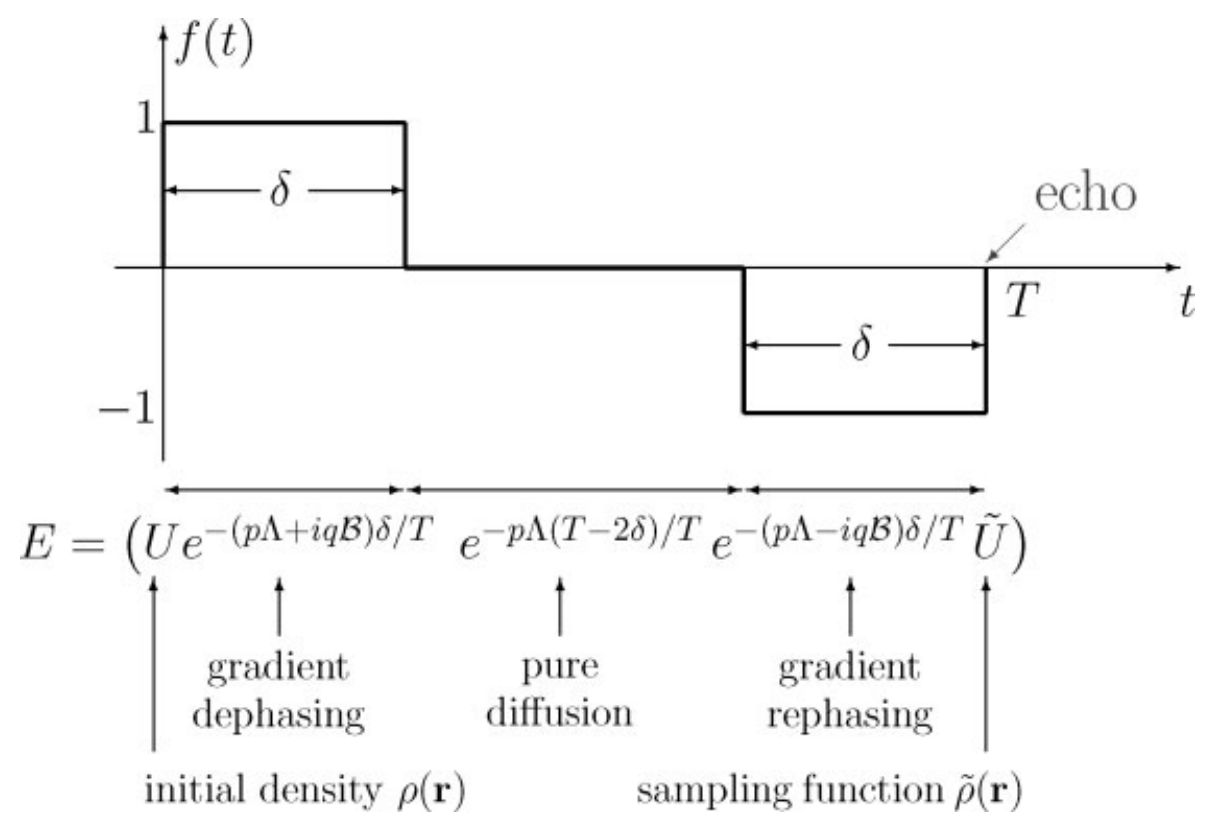

Figure 5 Stejskal-Tanner two-pulse profile $f(t)$ and the matrices describing the evolution of the magnetization on each subinterval with a constant gradient. This "matrix product rule" can be applied for any piecewise-constant profile, the resulting expression being composed of such elementary matrix blocks. Two vectors $U$ and $\tilde{U}$ determine the initial and final conditions. 
ond one starts and ceases at $t=T-\delta$ and $t=T$, respectively. The time interval $[0, T]$ is split in three subintervals on which the gradient is constant: $g$ on $[0, \delta], 0$ on $[\delta, T-\delta]$ (no gradient), and $-g$ on $[T-\delta, T]$. On each subinterval, the Bloch-Torrey equation can be solved using the above matrix exponential. To merge the solutions, the ending magnetization of the subinterval $k$ is used as an initial condition for solving the problem on the next subinterval $k+1$. The use of the Laplacian eigenfunctions and the consequent matrix representation is a particularly efficient way to handle such successive computations. So, the magnetization after the first gradient pulse (i.e., at the end of the first subinterval $[0, \delta]$ ) is determined by Eq. [25] with $t=\delta$. The vector $C(\delta)$, representing the magnetization $\mathrm{m}(\mathbf{r}, \delta)$ in the eigenbasis, is then used instead of $U$ as the initial condition for the next subinterval $[\delta, T-\delta]$. Because no gradient is applied there ( $g=0$ or $q=0$ ), the magnetization evolves in time according to the evolution operator $e^{-p \Lambda t / T}$ with $t=T-2 \delta$. At the end of this subinterval, the magnetization is represented as

$$
C(\delta) e^{-p \Lambda(T-2 \delta) / T}=U e^{-(p \Lambda+i q \mathcal{B}) \delta / T} e^{-p \Lambda(T-2 \delta) / T} .
$$

This vector is again used as the initial condition for the last subinterval $[T-\delta, T]$. The macroscopic signal at the echo time $t=T$ is then

$$
E=\left(U e^{-(p \Lambda+i q \mathcal{B}) \delta / T} e^{-p \Lambda(T-2 \delta) / T} e^{-(p \Lambda-i q \mathcal{B}) \delta / T} \tilde{U}\right)
$$

the matrices being applied from the left to the right. This "matrix product rule" can be applied for any piecewise-constant temporal profile $f(t)$ of the magnetic field. For example, for a CPMG sequence, formed by repetition of the above two-pulse profile, the amplitude of the $n$th echo is simply

$E_{n}=\left(U\left[e^{-(p \Lambda+i q \mathcal{B}) \delta / T} e^{-p \Lambda(T-2 \delta) / T} e^{-(p \Lambda-i q \mathcal{B}) \delta / T}\right]^{n} \tilde{U}\right)$.

A spectral analysis of the multiple echo attenuation for CPMG sequences was developed in (39).

In general, if $f(t)=f_{k}$ on the interval $\left[t_{k}, t_{k+1}\right]$ for $k$ ranging from 0 to $K$ (with $t_{0}=0$ and $t_{K+1}=T$ ), the macroscopic signal is

$$
E=\left(U\left[\prod_{k=0}^{K} e^{-\left(p \Lambda+i q f_{k} \mathcal{B}\right)\left(t_{k+1}-t_{k}\right) / T}\right] \tilde{U}\right) .
$$

Here, as previously, one successively applies from the left to the right the evolution operators $e^{-\left(p \Lambda+i q f_{k} \mathcal{B}\right)\left(t_{k+1}-t_{k}\right) / T}$. We stress that this result is exact, no approximation was involved. It is worth recalling that the matrices $\mathcal{B}$ and $\Lambda$ do not commute, so that the product in Eq. [32] cannot be reduced to a sum.

\section{Arbitrary Temporal Profiles}

This analysis can be applied to study complicated sequences of gradient pulses for gradient-echo, spinecho, or stimulated spin-echo experiments. Moreover, because any function can be approximated by a piecewise-constant function, the above relation allows one to approximately compute the signal for arbitrary temporal profile $f(t)$. As illustrated in Fig. 2(b), the time interval $[0, T]$ is divided into a large number $K$ of subintervals of equal durations $\tau=1 / K$ (in some cases, adaptive durations may be more efficient). On the $k^{\text {th }}$ subinterval, the function $f(t)$ is approximated by a constant $f_{k}=f(k \tau)$. For this piecewise-constant approximation, one can apply Eq. [33] with $t_{k+1}-t_{k}=\tau$

$$
E \simeq\left(U\left[\prod_{k=0}^{K} e^{-\left(p \Lambda+i q f_{k} \mathcal{B}\right) \tau / T}\right] \tilde{U}\right)
$$

We shall illustrate how easily this technique can be implemented in practice.

\section{Relaxation Mechanisms}

Up to this moment, we focused on the signal attenuation due to incomplete rephasing of the diffusing nuclei. In practice, however, various relaxation mechanisms may enhance the signal attenuation. For instance, paramagnetic oxygen molecules in the lungs lead to the bulk relaxation for MRI with hyperpolarized gases, while the iron in hemoglobin of the blood flowing through microcapillaries may cause surface relaxation on the alveolar membranes. The theoretical and numerical studies of these attenuation mechanisms were mainly limited to a uniform distribution of relaxing agents in the bulk or on the surface.

Bulk and Surface Relaxation. The case of uniform bulk relaxation is particularly simple because any diffusing particle can lose its magnetization with a constant relaxation rate, independently of its position and trajectory. In other words, uniform bulk relaxation affects all the particles in the same way. In practice, one usually compares the gradient-attenuated signal to a reference signal (without applied gradient). Because the bulk relaxation mechanism is independent of the gradient dephasing/rephasing, the reference signal is affected only by the former relaxa- 
tion mechanism, and its effect can be easily subtracted.

A uniform surface relaxation is a more complex attenuation mechanism because it affects only the diffusing particles in a close vicinity of the surface. Consequently, the relaxation of the diffusing particles depends on their individual trajectories. In fact, a particle which is started near the boundary has more chances to lose its magnetization than a particle which is started far from the boundary, especially when the diffusion time is short. For longer diffusion times, this difference is getting reduced because all the particles have enough time to explore the whole confining domain and to experience more or less the same relaxing interaction.

The usual way to include a uniform surface relaxation is to consider the Robin boundary condition [10] for the magnetization and, consequently, for the Laplace operator eigenfunctions. In particular, the above matrix formalism is directly applicable in the presence of uniform surface relaxation (1). But, the obvious inconvenience of this approach is that the eigenfunctions and eigenvalues depend on the dimensionless surface relaxivity $h$ in a complicated way (see Fig. 3). To study the effect of surface relaxation, one needs to recalculate these spectral characteristics of the Laplace operator and the governing matrices $\mathcal{B}$ and $\Lambda$ for each value of the surface relaxivity. Bearing in mind that the numerical computation of the eigenfunctions is a time-consuming task, this approach may appear less attractive than conventional numerical schemes. In contrast to the explicit dependence on the parameters $p$ and $q$ in Eqs. [29] and [31-33], the implicit dependence on the dimensionless surface relaxivity $h$ can be considered a drawback of this technique.

Alternative Insight. In a recent paper, we proposed an alternative solution to this problem (40). The idea is the following. Let us consider again the BlochTorrey equation [19] and put the Neumann boundary condition. In this equation, the last purely imaginary term was responsible for dephasing of the nuclei. If this term was real (with a coefficient $\kappa$ instead of $i q$ ), it would correspond to a pure relaxation mechanism with a given distribution $\kappa \tilde{B}(\mathbf{r})$ of the relaxation rates in the bulk. The relaxed signal has the form [29] where $i q \mathcal{B}$ is replaced by $\kappa \tilde{\mathcal{B}}$, with

$$
\tilde{\mathcal{B}}_{m, m^{\prime}}=\int_{\Omega} d \mathbf{r} u_{m}^{*}(\mathbf{r}) \tilde{B}(\mathbf{r}) u_{m^{\prime}}(\mathbf{r}) .
$$

The case of the uniform bulk relaxation corresponds to $\tilde{B}(\mathbf{r})=1$, so that $\tilde{\mathcal{B}}$ is simply the identity matrix

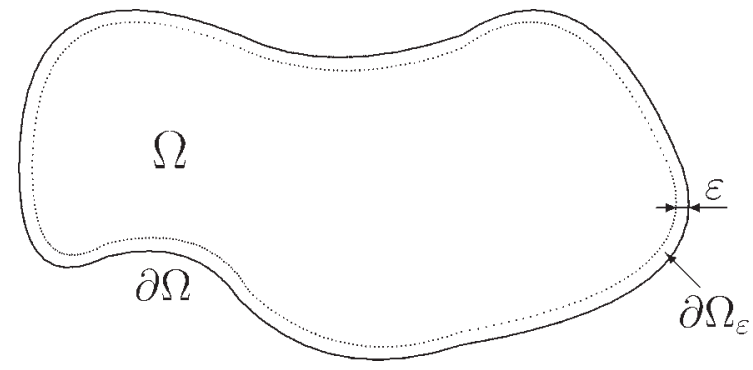

Figure 6 An $\varepsilon$-vicinity $\partial \Omega_{\varepsilon}$ of the boundary $\partial \Omega$ of a confining domain $\Omega$ is constituted of the points in $\Omega$ within the distance to the boundary smaller than $\varepsilon$. The $\varepsilon$-vicinity is introduced to account for the spins approaching to the boundary close enough to be affected by surface relaxation.

due to the normalization [15]: $\tilde{\mathcal{B}}_{m, m^{\prime}}=\delta_{m, m^{\prime}}$. Because the identity matrix commutes to any matrix, the contribution of the uniform bulk relaxation can be factored out. In general, a nonuniform distribution $\tilde{B}(\mathbf{r})$ can be used. In particular, if there is a specific relaxing subregion $A$ of the confining domain $\Omega$, one can take $\tilde{B}(\mathbf{r})=\mathbb{I}_{A}(\mathbf{r})$, where $\mathbb{I}_{A}(\mathbf{r})$ is the indicator function of this subregion: $\mathbb{I}_{A}(\mathbf{r})=1$ for $\mathbf{r} \in A$, and 0 otherwise.

\section{Alternative Implementation of Uniform Surface} Relaxation. In the case of uniform surface relaxation, a nucleus can lose its magnetization after hitting the boundary. This situation can be implemented via a distribution $\tilde{B}(\mathbf{r})$ localized near the boundary, e.g., $\tilde{B}(\mathbf{r})=\mathbb{I}_{\partial \Omega_{\varepsilon}}(\mathbf{r}) / \varepsilon$, where $\partial \Omega_{\varepsilon}$ is an $\varepsilon$-vicinity of the boundary: $\partial \Omega_{\varepsilon}=\{\mathbf{r} \in \Omega:|\mathbf{r}-\partial \Omega| \leq \varepsilon\}$ (see Fig. 6 ). In the limit $\varepsilon$ going to 0 , the volume integral over $\partial \Omega_{\varepsilon}$ in Eq. [34] is reduced to the boundary integral:

$$
\tilde{\mathcal{B}}_{m, m^{\prime}}^{s}=\int_{\partial \Omega} d \mathbf{r} u_{m}^{*}(\mathbf{r}) u_{m^{\prime}}(\mathbf{r}) .
$$

The parameter $\kappa$ is simply the product $p h=K T / L$. It is worth noting that both parameters $p$ and $h$ depend on the diffusion coefficient $D$, while the product does not. A nonuniform distribution of the relaxing agents on the boundary could be directly incorporated in this formula. Note that $\tilde{\mathcal{B}}^{s}$ is not an identity matrix (as it was for uniform bulk relaxation) because the normalization condition [15] says nothing about the boundary values of the eigenfunctions.

Because the mechanisms of gradient encoding and of bulk and surface relaxations are independent, their effects are simply superimposed as a linear combination of the corresponding terms in the Bloch-Torrey equation. Consequently, the above mentioned expres- 
sions for the signal can be easily modified to include different attenuation mechanisms. For instance, Eq. [29] for the FID signal in the presence of surface relaxation becomes

$$
E=\left(U e^{-\left(p \Lambda+i q \mathcal{B}+p h \tilde{\mathcal{B}}^{s}\right) t / T} \tilde{U}\right)
$$

The advantage of this relation is the explicit dependence on all three physical parameters $p, q$, and $h$. The structure of each term has a clear physical interpretation: $p \Lambda$ describes restricted diffusion, iq $\mathcal{B}$ represents the dephasing, and $p h \tilde{\mathcal{B}}^{s}$ accounts for surface relaxation. Similarly, the formula [30] for the signal attenuation by bipolar gradient shown in Fig. 5 takes the form

$$
\begin{array}{r}
E=\left(U e^{-\left(p \Lambda+i q \mathcal{B}+p h \tilde{\mathcal{B}}^{s}\right) \delta / T} e^{-\left(p \Lambda+p h \tilde{\mathcal{B}}^{s}\right)(T-2 \delta) / T}\right. \\
\left.e^{-\left(p \Lambda-i q \mathcal{B}+p h \tilde{\mathcal{B}}^{s}\right) \delta / T} \tilde{U}\right) .
\end{array}
$$

Since surface relaxation is present during the whole experiment (from the $90^{\circ} \mathrm{rf}$ pulse at $t=0 \mathrm{up}$ to the echo time $t=T$ ), the matrix $\tilde{\mathcal{B}}^{s}$ is included in each exponential. One can also extend Eqs. [31] and [32] for the CPMG sequence or any temporal profile. A nonuniform bulk relaxation can be similarly incorporated.

It is crucial to stress that here the eigenvalues and eigenfunctions are defined for the Neumann boundary condition, whatever the value of surface relaxivity. As a consequence, these eigenfunctions, as well as the governing matrices $\Lambda, \mathcal{B}$, and $\tilde{\mathcal{B}}^{s}$, depend only on the confining geometry and have to be constructed only once for a given confinement. The matrix $\tilde{\mathcal{B}}^{s}$ is an alternative way to introduce a uniform surface relaxation. Moreover, it is a general frame for dealing with a nonuniform distribution $\tilde{B}(\mathbf{r})$ of the relaxation rates, either in the bulk, or on the boundary. In this case, the matrix $\tilde{\mathcal{B}}$ would obviously depend on this distribution (as the matrix $\mathcal{B}$ depends on the spatial profile of the magnetic field). This concept is easily extendable for a superposition of various attenuation mechanisms. For instance, one can study the combined effect of the surface and bulk relaxations, gradient encoding, presence of dipolar magnetic field, etc.

\section{Numerical Implementation}

The practical efficiency of this numerical technique is based upon an unbounded increase of the eigenvalues $\lambda_{m}$ with $m$ : the matrix $\Lambda$ standing in the argument of the exponential function allows one to truncate the infinite-dimensional matrices $\Lambda, \mathcal{B}$, and $\tilde{\mathcal{B}}^{s}$ to moderate sizes. A very rough estimate of the truncation size $M$ could be given by the inequality

$$
p \lambda_{M} \gg q
$$

when "damping" real part $p \Lambda$ of the exponential function in Eq. [29] or similar expressions dominates its "oscillating" imaginary part iqB . Consequently, the matrices $\Lambda$ and $\mathcal{B}$ can be truncated to smaller sizes for smaller $q$ and larger $p$. We shall return to this question in Section "Choice of the Truncation Size".

The presented matrix formalism is a general mathematical frame for computing the signal attenuation because of restricted diffusion. On the basis of the Laplacian eigenfunctions, this approach can in principle be applied for any (bounded) geometrical confinement. In the next Section, we illustrate how this technique is implemented for rotation-invariant domains for which the matrices $\Lambda, \mathcal{B}$, and $\tilde{\mathcal{B}}^{s}$ are explicitly known (1). When the geometry of the confining domain $\Omega$ is more complex, one needs first to compute the eigenbasis of the Laplace operator in order to build the governing matrices. This is the most difficult and time-consuming step, but this computation should be performed only once for a given geometry. This is the crucial advantage of this numerical technique in comparison to conventional simulation schemes. When the preliminary construction of the governing matrices is done, the computation of the macroscopic signal for any set of physical parameters (free diffusion coefficient, gradient intensity, size of the confining domain, echo time) and any temporal profile $f(t)$ is straightforward, accurate, and rapid.

\section{PRACTICAL IMPLEMENTATION FOR SIMPLE DOMAINS}

In this Section, we illustrate how the numerical tool described in this article works for three simple domains: an interval, a disk, and a sphere. In these geometries, the Laplace operator eigenbasis is known analytically. For this reason, these three domains are often considered as "model geometries," on which various aspects of restricted diffusion can be thoroughly investigated. In fact, almost every quantity of interest can be computed analytically in this case ( 1 , $41)$. Note that analytical computations can also be performed for circular and spherical layers or shells (42). The computational code has been implemented by the author as a set of Matlab functions which are freely available on the web (43). 


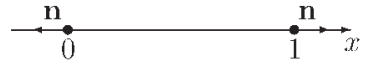

(1D)

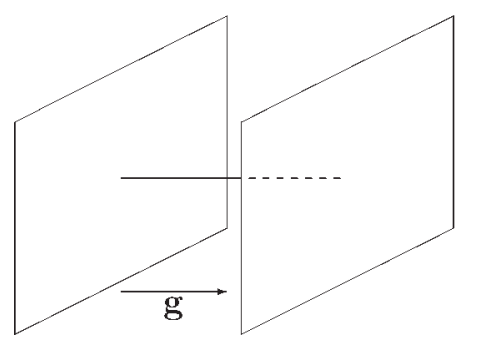

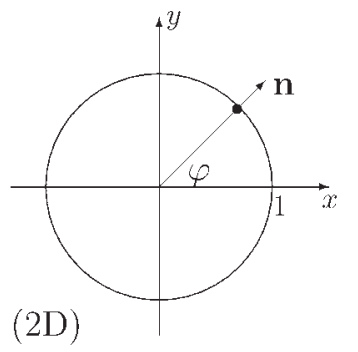

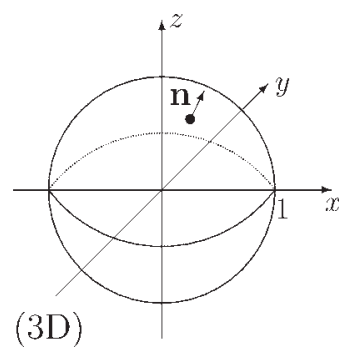

(3D)

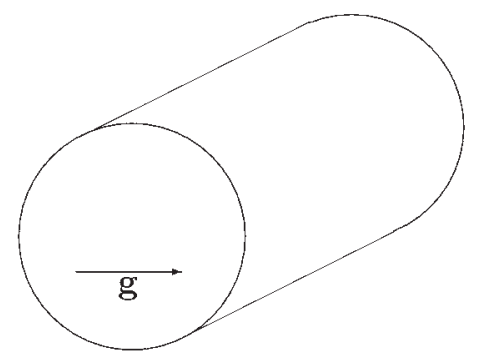

Figure 7 Three domains, for which the Laplacian eigenfunctions are known analytically: an interval (1D), a disk (2D), and a sphere (3D). The results for the interval and the disk are directly applicable for restricted diffusion in a slab (two parallel planes) and a cylinder, when the gradient is applied perpendicular to the planes or to the cylinder axis.

\section{The Unit Interval}

Eigenfunctions. We first consider restricted diffusion on the unit interval. In this case, a general form of a real-valued function satisfying Eq. [14a] is

$$
u(x)=c_{1} \cos (\alpha x)+c_{2} \sin (\alpha x),
$$

where $\alpha, c_{1}$, and $c_{2}$ are the three real numbers to be fixed by Eqs. [14b] and [15]. In fact, this function remains unchanged under the action of the Laplace operator $\Delta=d^{2} / d x^{2}$, up to a multiplicative factor $\alpha^{2}$ which can be recognized as the eigenvalue.

As shown in Section "Relaxation Mechanisms", the effect of surface relaxation can be incorporated by adding the matrix $\tilde{\mathcal{B}}^{s}$. This "trick" allows us to focus on the Neumann boundary condition instead of a more general Robin boundary condition [14b]. The boundary of the unit interval is constituted of two end-points $x=0$ and $x=1$. The normal derivative $\partial / \partial n$ is simply $-d / d x$ at $x=0$ and $+d / d x$ at $x=1$. The sign is different since the normal derivative is directed outwards the domain (Fig. 7). The Neumann boundary condition simply states that

$$
u^{\prime}(0)=0, \quad u^{\prime}(1)=0 .
$$

The first equation implies that the coefficient $c_{2}$ must be zero, while the second equation, $\sin (\alpha)=0$, determines $\alpha$. This equation has an infinite set of nonnegative solutions: $\alpha_{m}=\pi m$, where $m$ is a nonnegative integer index: $m=0,1,2, \ldots$. The last remaining coefficient $c_{1}$ can be fixed by choosing the normalization [15]. The resulting eigenfunctions and eigenvalues are

$$
u_{m}(x)=\varepsilon_{m} \cos (\pi m x), \quad \lambda_{m}=\pi^{2} m^{2},
$$

where $\varepsilon_{m}$ are normalization constants: $\varepsilon_{m}=\sqrt{2}$ for $m>0$, and $\varepsilon_{0}=1$.

It is worth noting that the analysis for Robin boundary condition would be in fact very similar. In this case, the eigenfunctions would be linear combinations of sine and cosine functions, but the equation for $\alpha$ would be more complicated:

$$
2 h \alpha \cos (\alpha)=\left(\alpha^{2}-h^{2}\right) \sin (\alpha) .
$$

When $h>0$, there is no explicit form for $\alpha$ so that one would need to solve this equation numerically. Although this is a simple task in numerical analysis, this computation would have to be repeated for each value of $h$. Figure 3 illustrates how the corresponding eigenfunctions vary with $h$. But, it is more appropriate, conceptually and numerically, to have the eigenbasis which depends only on the geometry, and not on physical parameters like $h$. For this reason, we consider the Laplacian eigenbasis for the Neumann boundary condition as the unique geometry-dependent basis. 
The Governing Matrices. Once the Laplacian eigenfunctions are found, the construction of the matrices $\Lambda, \mathcal{B}$, and $\tilde{\mathcal{B}}^{s}$ is straightforward. In fact, $\Lambda$ is simply a diagonal matrix constituted of the eigenvalues $\lambda_{m}=\pi^{2} m^{2}$. The matrix $\mathcal{B}$ depends on the choice of the spatial profile of the magnetic field. For a linear magnetic field gradient, the dimensionless spatial profile is $B(x)=x$, for which $(36,37)$ :

$$
\mathcal{B}_{m, m^{\prime}}=\varepsilon_{m} \varepsilon_{m^{\prime}} \int_{0}^{1} d x \cos (\pi m x) x \cos \left(\pi m^{\prime} x\right) .
$$

This integral is easy to calculate (see Table 1). The elements of the matrix $\tilde{\mathcal{B}}^{s}$ determining the effect of surface relaxation are found similarly. The explicit formulas allow one to construct easily the matrices $\Lambda, \mathcal{B}$ and $\tilde{\mathcal{B}}^{s}$ of any required size (the effect of truncation size is discussed in Section "Choice of the Truncation Size"). Note also that, if the initial density $\rho(x)$ and sampling function $\tilde{\rho}(x)$ are uniform, one has $U_{m}=\tilde{U}_{m}=\delta_{m, 0}$ because the ground eigenfunction $u_{0}(x)$ is constant for the Neumann boundary condition (otherwise, the only remaining computation would be the integration of $\rho(x)$ and $\tilde{\rho}(x)$ with cosine functions according to Eqs. [26] and [28]). We have therefore all the "ingredients" needed to calculate the signal. We conclude that the problem of finding the signal attenuation due to restricted diffusion on the unit interval is fully solved for any set of parameters $p, q$, and $h$ and any temporal profile. From a practical point of view, this technique is much simpler than other matrix formalisms (31-35) because the governing matrices do not depend on physical parameters.

General Observations. Figure 8 illustrates generic features in the behavior of the macroscopic signal. We consider restricted diffusion of the nuclei on the unit interval (in a slab). A bipolar gradient (with two rectangular pulses of duration $\delta=T / 2$ ) is applied so that the temporal profile $f(t)$ is simply 1 for $0<t<T / 2$ and -1 for $T / 2<t<T$ (this is also equivalent to a spin-echo experiment in a constant gradient with the $180^{\circ} \mathrm{rf}$ pulse at time $T / 2$ ). The signal as a function of the dimensionless gradient intensity $q$ is calculated by Eq. [30] with $\delta=T / 2$. For sake of simplicity, the surface relaxation $h$ is set to 0 . The dimensionless diffusion coefficient $p$ takes three values, representative of different diffusion regimes.

First, one can observe a parabolic shape for all the curves at small $q$ values. This is the usual quadratic dependence of $\log E$ on the gradient intensity, which is a reminiscent feature of free diffusion (cf. Eq.

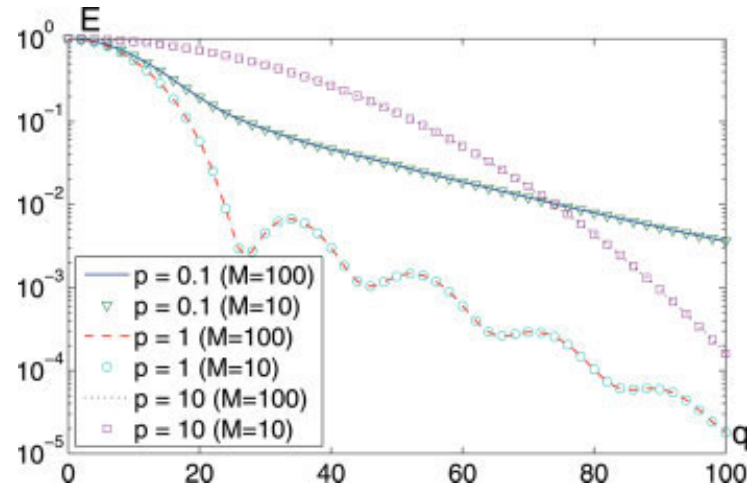

Figure 8 The signal at the echo time $t=T$ attenuated in a bipolar gradient due to restricted diffusion on the unit interval (a slab). The signal is plotted as a function of the dimensionless gradient intensity $q=\gamma g T L$ for three values of the dimensionless diffusion coefficient $p=D T / L^{2}$. The surface relaxivity $h$ is set to 0 . The curves computed with two truncation sizes, $M=10$ and $M=100$, are visually indistinguishable (the maximum relative errors at $q=$ 100 are $0.2 \%, 0.01 \%$, and $0.0007 \%$ for $p$ values $0.1,1$, and 10, respectively). This observation justifies the use of relatively small matrices for this range of physical parameters. [Color figure can be viewed in the online issue, which is available at www.interscience.wiley.com.]

[11]). For larger $q$, the presence of a geometrical confinement becomes significant, failing the Gaussian behavior. For slow diffusion $(p=0.1)$, the nuclei in the bulk far from the boundary are almost not restricted, so that one can formally apply Eq. [11] to show that their contribution to the signal is negligible at large gradients. The signal is then formed by the nuclei near the boundary which are more restricted and thus less dephased. This is known as the localization regime for which $\log E$ behaves asymptotically as $q^{2 / 3}(47-49)$. For the intermediate regime $(p=1)$, one can notice a kind of oscillatory behavior, although the temporal profile used is far from conventional narrow pulses and the underlying diffraction patterns (50-54). Finally, for $p=10$, the nuclei move rapidly enough to experience the dephasing by different gradients over the sample so that the net dephasing is somehow motionally averaged $(28,29)$. These and other theoretical issues were discussed at length in (l) and will be illustrated in a companion paper (2).

\section{Disk and Sphere}

Many analytical results can also be derived for a disk and a sphere. The rotation invariance of these 
Table 2 First 20 Positive Solutions of Eq. [39] and [40] in Ascending Order that Determine the Eigenvalues of the Laplace Operator with the Neumann Boundary Condition in the Unit Disk and the Unit Sphere

\begin{tabular}{lccccc}
\hline Disk & $1.841184 \alpha_{10}$ & $3.054237 \alpha_{20}$ & $3.831706 \alpha_{01}$ & $4.201189 \alpha_{30}$ & $5.317553 \alpha_{40}$ \\
& $5.331443 \alpha_{11}$ & $6.415616 \alpha_{50}$ & $6.706133 \alpha_{21}$ & $7.015587 \alpha_{02}$ & $7.501266 \alpha_{60}$ \\
& $8.015237 \alpha_{31}$ & $8.536316 \alpha_{12}$ & $8.577836 \alpha_{70}$ & $9.282396 \alpha_{41}$ & $9.647422 \alpha_{80}$ \\
\multirow{3}{*}{ Sphere } & $9.969468 \alpha_{22}$ & $10.17347 \alpha_{03}$ & $10.51986 \alpha_{51}$ & $10.71143 \alpha_{90}$ & $11.34592 \alpha_{32}$ \\
& $2.081576 \alpha_{10}$ & $3.342094 \alpha_{20}$ & $4.493409 \alpha_{01}$ & $4.514100 \alpha_{30}$ & $5.646704 \alpha_{40}$ \\
& $5.940370 \alpha_{11}$ & $6.756456 \alpha_{50}$ & $7.289932 \alpha_{21}$ & $7.725252 \alpha_{02}$ & $7.851078 \alpha_{60}$ \\
& $8.583755 \alpha_{31}$ & $8.934839 \alpha_{70}$ & $9.205840 \alpha_{12}$ & $9.840446 \alpha_{41}$ & $10.01037 \alpha_{80}$ \\
& $10.61386 \alpha_{22}$ & $10.90412 \alpha_{03}$ & $11.07021 \alpha_{51}$ & $11.07942 \alpha_{90}$ & $11.97273 \alpha_{32}$ \\
\hline
\end{tabular}

The smallest solution $\alpha_{00}=0$ is not shown.

domains allows one to rewrite Eqs. [14a] and [14b] in polar or spherical coordinates, when the radial and angular variables become separated (44-46). As earlier, Eq. [14a] determines a general analytical representation of the eigenfunctions, while Eq. [14b] "chooses" the appropriate form. For the unit disk $(L=1)$, one gets $(1)$

$$
u_{n k}(r, \varphi)=\left(\frac{\varepsilon_{n}}{\sqrt{\pi}} \frac{\beta_{n k}}{J_{n}\left(\alpha_{n k}\right)}\right) J_{n}\left(\alpha_{n k} r\right) \cos (n \varphi),
$$

where the factor in parentheses is a normalization constant (the coefficients $\beta_{n k}$ are given in Table 1), $J_{n}(z)$ the Bessel functions of the first kind, and $\alpha_{n k}$ all the nonnegative solutions of the equations

$$
J_{n}^{\prime}\left(\alpha_{n k}\right)=0 \quad(n=0,1,2,3 \ldots),
$$

representing the Neumann boundary condition. In particular, these solutions determine the eigenvalues: $\lambda_{n k}=\alpha_{n k}^{2}$.

There are many apparent differences with respect to the case of the unit interval:

(1) The radial dependence is determined by "special" Bessel functions instead of "elementary" cosine functions in Eq. [37]. However, the classical distinction between "special" and "elementary" functions is elusive and artificial: Bessel functions are as well studied and understood as cosine functions (55);

(2) The single equation $\sin (\alpha)=0$ for the unit interval is now replaced by a sequence of equations [39] enumerated by the nonnegative index $n=0,1,2,3 \ldots$ In turn, each of these equations has an infinite set of nonnegative solutions $\alpha_{n k}$, enumerated by the nonnegative index $k=0,1,2,3 \ldots$. For this reason, we use a double index $n k$ to distinguish different equations (index $n$ ) and different solutions (index $k$ ). But this double index plays exactly the same role as the single index $m$ for the unit interval. In fact, if all the solutions of all the equations [39] are sorted in ascending order, the position of the solution $\alpha_{n k}$ can serve as a single index $m$ :

$$
\begin{array}{ccccc}
k & 0 & 1 & 2 & \ldots \\
\alpha_{0 k} & 0.000 & 3.832 & 7.016 & \ldots \\
\alpha_{1 k} & 1.841 & 5.331 & 8.536 & \ldots \\
\alpha_{2 k} & 3.054 & 6.706 & 9.970 & \ldots \\
\alpha_{3 k} & 4.201 & 8.015 & 11.35 & \ldots \\
\ldots & \ldots & \ldots & \ldots & \ldots
\end{array}
$$

$$
\Downarrow
$$

$\begin{array}{cccccc}(n k) & 00 & 10 & 20 & 01 & \cdots \\ \alpha & 0.000 & 1.841 & 3.054 & 3.832 & \cdots \\ (m) & 0 & 1 & 2 & 3 & \cdots\end{array}$

It is clear that the enumeration of the eigenvalues and eigenfunctions can be equivalently performed either by the double index $n k$, or by the single index $m$. In what follows, we prefer to keep using the double index as a useful notation. For instance, the elements of the matrix $\Lambda$ will be written as $\Lambda_{n k, n^{\prime} k^{\prime}}$ instead of $\Lambda_{m, m^{\prime}}$. We hope that such double-index notation does not lead to a confusion (in particular, $\Lambda_{n k, n^{\prime} k^{\prime}}$ has nothing to do with a fourth rank tensor).

(3) There is no explicit formula for the solutions $\alpha_{n k}$ of Eq. [39], so that these equations have to be solved numerically. This is a simple task in numerical analysis. Moreover, these solutions have to be found only once so that this preliminary step does not complicate further computation of the signal. Table 2 contains the first twenty positive solutions in ascending order which are sufficient for many practical purposes.

The knowledge of the eigenfunctions allows one to construct the governing matrices $\Lambda, \mathcal{B}$, and $\tilde{\mathcal{B}}^{s}$. However, the computation of the matrix $\mathcal{B}$ for a linear magnetic field gradient $B(\mathbf{r})=r \cos \varphi$ requires 
sophisticated integration with Bessel functions. The explicit formulas for its elements, which were recently obtained in $(1,43)$, are reproduced for the Neumann boundary condition in Table 1 . In summary, the original problem of finding the signal attenuation due to restricted diffusion is fully reduced to the numerical computation of the nonnegative solutions of Eq. [39].

A similar analysis is carried out for the unit sphere, for which the eigenfunctions can be written as

$$
u_{n k l}(r, \theta, \varphi)=\left(\frac{\beta_{n k}}{\sqrt{2 \pi} j_{n}\left(\alpha_{n k}\right)}\right) j_{n}\left(\alpha_{n k} r\right) P_{n}(\cos \theta) e^{i l \varphi}
$$

where the factor in parentheses is a normalization constant (the coefficients $\beta_{n k}$ are given in Table 1), $j_{n}(z)$ the spherical Bessel functions of the first kind, $j_{n}(z)=\sqrt{\frac{\pi}{2 z}} J_{n+1 / 2}(z)$, and $\alpha_{n k}$ all the nonnegative solutions of the equations

$$
j_{n}^{\prime}\left(\alpha_{n k}\right)=0 \quad(n=0,1,2,3 \ldots),
$$

representing the Neumann boundary condition. In particular, these solutions determine the eigenvalues: $\lambda_{n k}=\alpha_{n k}^{2}$. Note that for a linear magnetic field gradient $B(\mathbf{r})=r \cos \theta$, there is no dependence on $\varphi$, so that the third index $l$ of the eigenfunctions is irrelevant, and it can be omitted. Table 1 summarizes the main formulas required to construct the matrices $\Lambda$, $\mathcal{B}$, and $\tilde{\mathcal{B}}^{s}$. As earlier, the computation is reduced to finding the solutions of Eq. [40]. Its first 20 positive solutions in ascending order are tabulated in Table 2.

\section{Choice of the Truncation Size}

The choice of the appropriate values for numerical parameters is a compromise between accuracy and rapidity. For the matrix technique, the crucial parameter to choose is the truncation size $M$ of the governing matrices. In fact, the set of Laplacian eigenfunctions is infinite, and each of them contributes to the signal. Consequently, the compact form [29] or similar expressions are exact only when the governing matrices are infinite-dimensional. Even for the unit interval, for which the elements of these matrices are known explicitly, the practical computation requires a truncation to finite sizes, being thus a source of computational errors.

There is no universal criterion for choosing the truncation size $M$. At the same time, one can follow a simple empirical principle that the obtained results should not (almost) change when $M$ is further increased. In practice, it is advised to calculate the signal twice, by using the matrices truncated to a size $M$ and to a larger size $M^{\prime}$. The difference between two results indicates the order of truncation error. This principle is illustrated in Fig. 8. The three curves, obtained by using the governing matrices $\mathcal{B}$ and $\Lambda$ truncated to $M=10$ and $M^{\prime}=100$, are visually indistinguishable. The corresponding relative errors are maximum at $q=100$, their values being $0.2 \%, 0.01 \%$, and $0.0007 \%$ for $p$ values $0.1,1$, and 10 , respectively. This observation is in agreement with our previous reasoning: for smaller $p$ and larger $q$, the term iqB in Eq. [30] is a stronger perturbation of $p \Lambda$, and the accuracy of truncation is poorer (but still excellent!). The fact that smaller $p$ values require larger number of Laplacian eigenfunctions can also be seen from the spectral decomposition [18] for the diffusive propagator. Note that the tenfold value of $M^{\prime}$ is willingly exaggerated, a smaller value $M^{\prime}=20$ would be sufficient to check the accuracy in this particular case. This remarkably good accuracy should not be surprising because a substantial part of computations had been performed analytically (exact formulas for the matrix $\mathcal{B}$ ).

Although Fig. 8 justifies the use of small governing matrices in the considered example (the truncation size $M=10$ was sufficient), this observation should not be blindly extended to any cases. As we already stated, the choice of the truncation size is an important step, and one has to check the accuracy in each particular situation. Let us mention at least two reasons which may require the use of (much) larger governing matrices.

Slower Increase of the Eigenvalues with Their Order Index m. According to Eq. [36], the accuracy of computation depends on whether the eigenvalue $\lambda_{M}$ is small or large with respect to $p$ and $q$. The choice of this value implies in turn the choice of the truncation size $M$. For the unit interval, the eigenvalues are simply related to their order index: $\lambda_{m}=\pi^{2} M^{2}$. In general, one can use the Weyl asymptotic law stating that $\lambda_{m} \propto M^{2 / d}$ in an embedding space of $d$ dimensions (56). This means that in order to attain the chosen value $\lambda_{M}$ (determining the accuracy), one needs to calculate $M \propto \lambda_{M}^{d / 2}$ eigenmodes. In practice, the computation would require larger matrices in higher dimensions. The analysis for the unit interval is therefore the simplest case. The computation for the disk $(d=2)$ is more time consuming because the eigenvalues grow only linearly with $M$, as one can notice from Table 2. For instance, the twentieth positive eigenvalue $\lambda_{32}=\alpha_{32}^{2} \approx 128.73$ is much smaller than the twentieth positive eigenvalue $\pi^{2} 20^{2} \approx 3947.84$ for the unit interval. When the 
required truncation size is not accessible, other methods can be used to improve the accuracy, e.g., an extrapolation of the signal as a function of the truncation size $M$ for $M$ going to infinity.

The computation would require still larger matrices in three dimensions. Note that the unit sphere is not representative because the eigenmodes in this exceptional case are degenerate so that the eigenvalues grow linearly with $M$, as for the disk. This degeneracy is related to rotation invariance of the sphere and will be broken for other three-dimensional shapes. Further discussion of this issue is beyond the scope of this paper.

In spite of these precautions, we emphasize that the matrix technique remains a very powerful numerical tool in two and three dimensions. In particular, its use for computing the signal in a disk and a sphere is much faster and more accurate than by using conventional Monte Carlo simulations.

\section{Introduction of Surface Relaxation via Matrix} $\tilde{\mathcal{B}}^{s}$. From our practical experience, the choice of the truncation size considerably depends on the dimensionless surface relaxation $h$. For the same accuracy, the computation for larger $h$ requires larger matrices. In fact, the effect of truncation is much more pronounced for the matrix $\tilde{\mathcal{B}}^{s}$ than for the matrix $\mathcal{B}$. This is not surprising: from Table 1 , the nonzero elements of the matrix $\widetilde{\mathcal{B}^{s}}$ are of the same order of magnitude, while the elements of the matrix $\mathcal{B}$ progressively decrease with $\left|m-m^{\prime}\right|$ (when one moves away from the diagonal). Qualitatively, the matrix $\tilde{\mathcal{B}}^{s}$ is a "stronger" perturbation of the matrix $\Lambda$. If $\Lambda$ was infinite-dimensional, this effect would be irrelevant. In practice, larger finite-dimensional matrices are needed to diminish this effect. When the surface relaxation is very high (in particular, in the limit of the Dirichlet boundary condition when $h=\infty$ ), the use of the matrix $\tilde{\mathcal{B}}^{s}$ is not appropriate. In this case, one can still apply the matrix technique, but the computation of Laplacian eigenfunctions with Robin (or Dirichlet) boundary condition is preferred. The corresponding explicit formulas for computing the matrices $\Lambda$ and $\mathcal{B}$ for the interval, disk, and sphere are given in $(1)$. On the other hand, it is worth noting that very high surface relaxivity is not typical for NMR applications. The formulas presented in this paper are therefore applicable for most cases of practical interest. A systematic study of these issues would be helpful.

\section{Temporal Profiles and Time Step}

Except for particular situations (e.g., bipolar gradient pulses of rectangular shape shown in Fig. 5), a given temporal profile $f(t)$ has to be approximated by a piecewise-constant function as illustrated in Fig. $2 \mathrm{~b}$. In this case, the discretization time step $\tau$ is another numerical parameter to choose. Once again, it is difficult to provide a universal recipe for this choice. Naturally, $\tau$ should be small as compared to relevant time scales of the temporal profile $f(t)$ (in particular, the ramp time and plateau duration for trapezoidal pulses). As for the truncation size, it is advised to make discretizations with two time steps $\tau$ and $\tau^{\prime}$, and to check for the obtained results to be close enough. As an example, we consider restricted diffusion on the unit interval and calculate the signal for different discretizations of the cosine profile $f(t)=\cos (\pi t / T)$. For $p=q=1, h=0$, and $M=50$, we empirically obtained $\left|E_{\tau}-E_{\tau=0}\right| \sim 0.8(\tau / T)^{2}$, where $E_{\tau}$ is the signal computed for the time step $\tau$, and $E_{\tau=0}$ its interpolated value at $\tau=0$. A moderate choice $\tau / T=0.01$ ensures an accuracy of order of $10^{-4}$ for this profile.

\section{Computational Efficiency}

Up to this moment, we addressed the accuracy of the matrix technique which has been shown to be remarkably good. Let us now briefly discuss its counterpart, the computational time (CPU). We are not intended to provide here neither extensive analysis for this characteristics, nor its careful comparison to other computational schemes such as Monte Carlo simulations or finite difference method. Although the computational time may depend on various parameters, we focus on the truncation size $M$ and discretization time step $\tau$.

Manipulations with matrices (e.g., inversion, finding eigenvectors, etc.) often require $O\left(M^{3}\right)$ operations so that the CPU is expected to grow as $M^{3}$. This behavior has been empirically checked for restricted diffusion on the unit interval with a simple bipolar gradient profile. The CPU for computing the signal at one point (a fixed set of $p, q$, and $h$ values) on a home laptop computer (Intel processor $1.73 \mathrm{GHz}$ ) was of the order of $3 \times 10^{-7} M^{3}$ s. Even for large $200 \times 200$ matrices, this computation takes only few seconds, and this characteristics can still be substantially improved by optimizing the matrix operations. Given that for the unit interval the accuracy was shown to be high even for $M=20$, the matrix technique is superior with respect to conventional simulation schemes, at least for simple confining domains.

When the temporal profile $f(t)$ has to be approximated by a piecewise-constant function, the signal is computed by matrix multiplication in Eq. [33]. The computational time is expected to grow linearly with 
the number of factors in this product which is proportional to $T / \tau$. This behavior has been empirically checked for restricted diffusion on the unit interval. For $p=q=1, h=0, M=50$, and the cosine temporal profile, we obtained that CPU was approximately $0.014(T / \tau)+0.42 \mathrm{~s}$. Even for $\tau=0.01 T$, the computation takes only few seconds, while the accuracy was shown to be better than $10^{-4}$. Note also that the truncation size $M$ could be reduced to get still faster computation.

In summary, the matrix technique turns out to be a powerful numerical tool to get very accurate results in a short computational time. For simple confining domains (slab, cylinder, and sphere), this technique is easy to implement, and it is highly recommended instead of using conventional simulation schemes (such as Monte Carlo simulations, finite difference or finite element methods).

\section{CONCLUSION}

It is quite surprising that matrix-like formalisms appeared in NMR literature only a decade ago. After all, the idea of using Laplacian eigenfunctions as natural "bricks" to build a theoretical basic for restricted diffusion is old and genuine. A compact expression [29] for the signal is merely a representation of the Bloch-Torrey equation in the Laplace operator eigenbasis. Moreover, this spectral description led us to an efficient numerical tool which is not being broadly employed yet. This is a simple tool, both from conceptual and numerical points of view. Each attenuation mechanism is represented by its own matrix depending only on the geometry of the confinement. In turn, the "strength" of each mechanism is determined by the corresponding physical parameter. In this way, the roles of physics and the geometry are somehow separated. Once the governing matrices are constructed for a given confinement, the computation of the signal for any (reasonable) set of physical parameters is easy, rapid, and accurate.

The matrix formalism involving Laplacian eigenfunctions is a general and well-adapted mathematical language to tackle various problems of restricted diffusion in NMR, at both theoretical and numerical levels. This language is very flexible and easily extendable. In particular, one can implement any spatial profile of the magnetic field, not only a linear gradient considered here. This implementation only requires to recalculate the matrix $\mathcal{B}$ with a given function $B(\mathbf{r})$ according to Eq. [22]. For instance, restricted diffusion in a parabolic magnetic field was investigated in (1), while the choice of the cosine magnetic field in a slab geometry led to a particularly simple structure of the matrix $\mathcal{B}$ (57). The effect of internal gradients due to magnetic susceptibility difference can also be analyzed [its relation to Laplacian eigenfunctions was explored by Song and coworkers (58-62)]. Similarly, nonuniform surface or bulk relaxation mechanisms can be directly incorporated via the matrices $\tilde{\mathcal{B}}^{s}$ and $\tilde{\mathcal{B}}$.

The computational problem is reduced to finding Laplacian eigenfunctions. For the unit interval and rotation-invariant domains (disk and sphere, as well as circular and spherical layers), for which the Laplace operator eigenbasis is known analytically, the matrix technique is definitely more accurate and much faster than conventional schemes such as Monte Carlo simulations. In sharp contrast with the conventional schemes, the computational time weakly depends on physical parameters, allowing for long-time or high-gradient analysis. For complex geometries, finding Laplacian eigenfunctions is a difficult but classical task in numerical analysis. Studying restricted diffusion via the Laplacian eigenfunctions in some model geometries (such as hierarchical morphologies, self-similar fractals, ordered or random packs of spherical beads, etc.) is a promising perspective for future research. In particular, the roles of multiple length scales, branching or interconnected morphologies, roughness of the boundary and many other geometrical features are intriguing from the theoretical point of view and crucial for practical implementations, ranging from oil recovery to medical applications.

\section{ACKNOWLEDGMENTS}

The author thanks Dr. G. Guillot for careful reading of the manuscript and fruitful discussions. The work has been supported by ANR grants "MIPOMODIM" and "DYOPTRI".

\section{REFERENCES}

1. Grebenkov DS. 2007. NMR survey of reflected Brownian motion. Rev Mod Phys 79:1077-1137.

2. Grebenkov DS. Laplacian eigenfunctions in NMR II. Theoretical advances. Concepts Magn Reson A, in preparation.

3. Freidlin M. 1985. Functional Integration and Partial Differential Equations. Annals of Mathematics Studies New Jersey, Princeton: Princeton University.

4. Klafter J, Drake JM, editors. 1989. Molecular Dynamics in Restricted Geometries. New York: Wiley. 
5. Bouchaud JP, Georges A. 1990. Anomalous diffusion in disordered media: Statistical mechanisms, models and physical applications. Phys Rep 195:127-293.

6. Shlesinger MF, Klafter J, Zumofen G. 1999. Above, below and beyond Brownian motion. Am J Phys 67:1253-1259.

7. Metzler R, Klafter J. 2000. The random walk's guide to anomalous diffusion: A fractional dynamics approach. Phys Rep 339:1-77.

8. Havlin S, ben Avraham D. 2002. Diffusion in disordered media. Adv Phys 51:187-292.

9. Kimmich R. 2002. Strange kinetics, porous media, and NMR. Chem Phys 284:253-285.

10. Feller W. 1971. An Introduction to Probability Theory and Its Applications, Vols. I, II, 2nd Ed. New York: Wiley.

11. Sapoval B. 1994. General formulation of Laplacian transfer across irregular surfaces. Phys Rev Lett 73:3314-3316.

12. Sapoval B. 1996. Transport across irregular interfaces: Fractal electrodes, membranes and catalysts. In: Bunde A, Havlin S, editors. Fractals and Disordered Systems, Berlin: Springer. pp 233-261.

13. Callaghan PT. 1991. Principles of Nuclear Magnetic Resonance Microscopy. Oxford: Clarendon.

14. Torrey HC. 1956. Bloch equations with diffusion terms. Phys Rev 104:563-565.

15. Zielinski LJ, Sen PN. 2003. Combined effects of diffusion, nonuniform-gradient magnetic fields, and restriction on an arbitrary coherence pathway. J Chem Phys 119:1093-1104.

16. Hahn EL. 1950. Spin Echoes. Phys Rev 80:580-594.

17. Carr HY, Purcell EM. 1954. Effects of diffusion on free precession in NMR experiments. Phys Rev 94:630-638.

18. Filoche M, Sapoval B. 1999. Can one hear the shape of an electrode? II. Theoretical study of the Laplacian transfer. Eur Phys J B 9:755-763.

19. Grebenkov DS, Filoche M, Sapoval B. 2003. Spectral properties of the Brownian self-transport operator. Eur Phys J B 36:221-231.

20. Grebenkov DS, Filoche M, Sapoval B, Felici M. 2005. Diffusion-reaction in branched structures Theory and application to the lung acinus. Phys Rev Lett 94:050602.

21. Felici M, Filoche M, Straus C, Similowski T, Sapoval B. 2005. Diffusional screening in real 3D human acini - A theoretical study. Resp Physiol Neurobiol 145:279-293.

22. Grebenkov DS, Filoche M, Sapoval B. 2006. Mathematical basis for a general theory of Laplacian transport towards irregular interfaces. Phys Rev E 73:021103.

23. Grebenkov DS. 2006. Partially reflected Brownian motion: A stochastic approach to transport phenomena. In: Velle LR, editor. Focus on Probability Theory. Hauppauge, NY: Nova Science Publishers. p. 135-169.
24. Grebenkov DS. 2006. Scaling Properties of the Spread Harmonic Measures. Fractals 14:231-243.

25. Jackson JD. 2006. Mathematics for Quantum Mechanics: An Introductory Survey of Operators, Eigenvalues, and Linear Vector Spaces. New York: Dover Publications.

26. Birman MS, Solomyak MZ. 1987. Spectral Theory of Self-Adjoint Operators in Hilbert Space. Dordrecht, The Netherlands: D. Reidel Publishing Company.

27. Ryu S. 2001. Probing pores using elementary quantum mechanics. Magn Reson Imaging 19:411-415.

28. Robertson B. 1966. Spin-echo decay of spins diffusion in a bounded region. Phys Rev 151:273277.

29. Neuman CH. 1974. Spin echo of spins diffusion in a bounded medium. J Chem Phys 60:4508-4511.

30. Brownstein KR, Tarr CE. 1979. Importance of classical diffusion in NMR studies of water in biological cells. Phys Rev A 19:2446-2453.

31. Caprihan A, Wang LZ, Fukushima E. 1996. A multiple-narrow-pulse approximation for restricted diffusion in a time-varying field gradient. J Magn Reson A 118:94-102.

32. Callaghan PT. 1997. A simple matrix formalism for spin echo analysis of restricted diffusion under generalized gradient waveforms. J Magn Reson 129: 74-84.

33. Callaghan PT, Codd SL. 1998. Generalized calculation of NMR imaging edge effects arising from restricted diffusion in porous media. Magn Reson Imag 16:471-478.

34. Codd SL, Callaghan PT. 1999. Spin echo analysis of restricted diffusion under generalized gradient waveforms: Planar, cylindrical and spherical pores with wall relaxivity. J Magn Reson 137:358-372.

35. Sukstanskii AL, Yablonskiy DA. 2002. Effects of restricted diffusion on MR signal formation. J Magn Reson 157:92-105.

36. Barzykin AV. 1998. Exact solution of the TorreyBloch equation for a spin echo in restricted geometries. Phys Rev B 58:14171-14174.

37. Barzykin AV. 1999. Theory of spin echo in restricted geometries under a step-wise gradient pulse sequence. J Magn Reson 139:342-353.

38. Axelrod S, Sen PN. 2001. Nuclear magnetic resonance spin echoes for restricted diffusion in an inhomogeneous field: Methods and asymptotic regimes. J Chem Phys 114:6878-6895.

39. Grebenkov DS. 2006. Multiexponential attenuation of the CPMG spin echoes due to a geometrical confinement. J Magn Reson 180:118-126.

40. Grebenkov DS. 2007. Residence times and other functionals of reflected Brownian motion. Phys Rev E 76:041139.

41. Grebenkov DS. 2007. Multiple correlation function approach: Rigorous results for simple geometries. Diff Fundam 5:1-34.

42. Grebenkov DS. 2008. Analytical solution for restricted diffusion in circular and spherical layers in 
inhomogeneous magnetic fields. J Chem Phys 128:134702.

43. Multiple Correlation Function Approach Library (Matlab code). Online: http://pmc.polytechnique.fr/ pagesperso/dg/MCF/MCF_e.htm. Put 2006.

44. Courant R, Hilbert D. 1989. Methods of Mathematical Physics, Vol. 1. New York: Wiley-VCH.

45. Carslaw HS, Jaeger JC. 1959. Conduction of Heat in Solids, 2nd Ed. Oxford: Clarendon.

46. Crank J. 1975. The Mathematics of Diffusion, 2nd Ed. Oxford: Clarendon.

47. Stoller SD, Happer W, Dyson FJ. 1991. Transverse spin relaxation in inhomogeneous magnetic fields. Phys Rev A 44:7459-7477.

48. de Swiet TM, Sen PN. 1994. Decay of nuclear magnetization by bounded diffusion in a constant field gradient. J Chem Phys 100:5597-5604.

49. Hürlimann MD, Helmer KG, de Swiet TM, Sen PN, Sotak CH. 1995. Spin echoes in a constant gradient and in the presence of simple restriction. J Magn Reson A 113:260-264.

50. Tanner JE, Stejskal EO. 1968. Restricted self-diffusion of protons in colloidal systems by the pulsedgradient, spin-echo method. J Chem Phys 49:1768-1777.

51. Callaghan PT, Coy A, MacGowan D, Packer KJ, Zelaya FO. 1991. Diffraction-like effects in NMR diffusion studies of fluids in porous solids. Nature (London) 351:467-469.

52. Price WS. 1997. Pulsed-field gradient nuclear magnetic resonance as a tool for studying translational diffusion. I. Basic theory. Concepts Magn Reson 9:299-336.

53. Price WS. 1998. Pulsed-field gradient nuclear magnetic resonance as a tool for studying translational diffusion. II. Experimental aspects. Concepts Magn Reson 10:197-237.

54. Callaghan PT, Codd SL, Seymour JD. 1999. Spatial coherence phenomena arising from translational spin motion in gradient spin echo experiments. Concepts Magn Reson 11:181-202.
55. Watson GN. 1962. A Treatise on the Theory of Bessel Functions. Cambridge: Cambridge University Press.

56. Lapidus ML. 1991. Fractal drum, inverse spectral problems for elliptic operators and a partial resolution of the Weyl-Berry conjecture. Trans Am Math Soc 325:465-529.

57. Grebenkov DS. 2007. NMR restricted diffusion between parallel planes in a cosine magnetic field: An exactly solvable model. J Chem Phys 126:104706.

58. Song YQ. 2000. Detection of the high eigenmodes of spin diffusion in porous media. Phys Rev Lett 85:3878-3881.

59. Song YQ, Ryu S, Sen PN. 2000. Determining multiple length scales in rocks. Nature (London) 406:178-181.

60. Lisitza NV, Song YQ. 2001. The behavior of diffusion eigenmodes in the presence of internal magnetic field in porous media. J Chem Phys 114:9120-9124.

61. Lisitza NV, Song YQ. 2002. Manipulation of the diffusion eigenmodes in porous media. Phys Rev B 65:172406

62. Song YQ. 2003. Using internal magnetic fields to obtain pore size distributions of porous media. Concepts Magn Reson A 18:97-110.

\section{BIOGRAPHY}

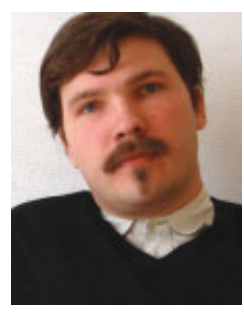

Denis S. Grebenkov has received two $\mathrm{PhD}$ degrees, the first in statistical physics from Saint Petersburg State University (Russia), in 2003; and the second in theoretical physics from Ecole Polytechnique (France), in 2004. He did postgraduate researches on lung imaging at University Paris-XI (France), and on nonequilibrium dynamics of granular media at University of Naples (Italy). In 2006, he got a permanent research position at Laboratory of Condensed Matter Physics, CNRS - Ecole Polytechnique (France), to investigate restricted diffusion in complex geometries. 